\title{
Integration of UPR ${ }^{\mathrm{ER}}$ and Oxidative Stress Signaling in the Control of Intestinal Stem Cell Proliferation
}

\section{Lifen Wang ${ }^{1}$, Xiankun Zeng ${ }^{2}$, Hyung Don Ryoo ${ }^{3}$, Heinrich Jasper ${ }^{1 *}$}

1 Buck Institute for Research on Aging, Novato, California, United States of America, 2 Basic Research Laboratory, Center for Cancer Research, National Cancer Institute, Frederick, Maryland, United States of America, 3 Department of Cell Biology, New York University School of Medicine, New York, New York, United States of America

\begin{abstract}
The Unfolded Protein Response of the endoplasmic reticulum (UPR ${ }^{\mathrm{ER}}$ ) controls proteostasis by adjusting the protein folding capacity of the ER to environmental and cell-intrinsic conditions. In metazoans, loss of proteostasis results in degenerative and proliferative diseases and cancers. The cellular and molecular mechanisms causing these phenotypes remain poorly understood. Here we show that the UPR ${ }^{\mathrm{ER}}$ is a critical regulator of intestinal stem cell (ISC) quiescence in Drosophila melanogaster. We find that ISCs require activation of the UPR ${ }^{\mathrm{ER}}$ for regenerative responses, but that a tissue-wide increase in ER stress triggers ISC hyperproliferation and epithelial dysplasia in aging animals. These effects are mediated by ISCspecific redox signaling through Jun-N-terminal Kinase (JNK) and the transcription factor CncC. Our results identify a signaling network of proteostatic and oxidative stress responses that regulates ISC function and regenerative homeostasis in the intestinal epithelium.
\end{abstract}

Citation: Wang L, Zeng X, Ryoo HD, Jasper H (2014) Integration of UPR ${ }^{\mathrm{ER}}$ and Oxidative Stress Signaling in the Control of Intestinal Stem Cell Proliferation. PLoS Genet 10(8): e1004568. doi:10.1371/journal.pgen.1004568

Editor: Norbert Perrimon, Harvard Medical School, Howard Hughes Medical Institute, United States of America

Received April 4, 2014; Accepted July 1, 2014; Published August 28, 2014

This is an open-access article, free of all copyright, and may be freely reproduced, distributed, transmitted, modified, built upon, or otherwise used by anyone for any lawful purpose. The work is made available under the Creative Commons CCO public domain dedication.

Data Availability: The authors confirm that all data underlying the findings are fully available without restriction. All relevant data are within the paper and its Supporting Information files.

Funding: This work was funded by the National Institute on Aging (R01 AG028127 to HJ), the National Institute on General Medical Sciences (R01 GM100196 to $\mathrm{HJ}$ ), the National Eye Institute (R01 EY020866 to HDR), and an Ellison Medical Foundation/AFAR postdoctoral Fellowship (LW). The funders had no role in study design, data collection and analysis, decision to publish, or preparation of the manuscript.

Competing Interests: The authors have declared that no competing interests exist.

*Email: hjasper@buckinstitute.org

\section{Introduction}

Long-term homeostasis of high-turnover tissues relies on the precise regulation of stem cell (SC) activity that allows tailoring regenerative responses to the needs of the tissue. Regenerative processes in barrier epithelia, such as the intestinal epithelium, are particularly vulnerable to exogenous insults. Understanding how cellular stress responses of intestinal epithelial cells (IECs) and intestinal stem cells (ISCs) coordinate and maintain regenerative processes in the gut will provide insight into the etiology of pathologies ranging from inflammatory bowel diseases (IBDs) to colorectal cancers.

The unfolded protein response of the ER $\left(\mathrm{UPR}^{\mathrm{ER}}\right)$ plays a central role in the control of homeostasis of the intestinal epithelium. Loss of protein folding capacity in the ER of IECs results in complex cell-autonomous and non-autonomous activation of stress signaling pathways, triggering an inflammatory condition that severely perturbs proliferative homeostasis, innate immune function and cell survival in the epithelium, and has been implicated in IBDs [1-7].

The $\mathrm{UPR}^{\mathrm{ER}}$ is triggered by the accumulation of misfolded proteins in the ER [8], which activate three highly conserved $\mathrm{UPR}^{\mathrm{ER}}$ sensors: the PKR-like ER kinase PERK, the transcription factor ATF6, and the endoribonuclease IRE1 (Figure 1B). These sensors make up the three branches of UPR ${ }^{\mathrm{ER}}$ signaling, which consists of IRE1-mediated splicing of the mRNA encoding the bZip transcription factor X-Box binding protein 1 (Xbpl), phosphorylation of the translation initiation factor 2 alpha (eIF2 $\alpha$ ) by PERK, and cleavage and activation of ATF6, resulting in its nuclear translocation and activation of stress response genes, including Xbpl [1-7,9]. Xbpl regulates transcription of ER components, and the resulting transcriptional induction of ER chaperones and of genes encoding ER components enhances ER folding capacity, and the reduction in protein synthesis (by eIF2 $\alpha$ ) alleviates the protein load in the ER. Furthermore, factors required to degrade un/misfolded proteins through ER-associated degradation (ERAD) are induced [8,10-12]. The accumulation of un/misfolded proteins in the ER is further associated with increased production of reactive oxygen species (ROS), most likely due to the production of hydrogen peroxide as a byproduct of protein disulfide bond formation by protein disulfide isomerase (PDI) and ER oxidoreductin 1 (Erol) [13-15].

Recent studies suggest that the UPR ${ }^{\mathrm{ER}}$ may influence regenerative processes in the gut directly, as it is engaged in cells transitioning from a stem-like state into the transit amplifying state in the small intestine of mice [16]. Regeneration is also influenced by the intracellular redox state of stem cells, and changes in intracellular ROS production play an important role in the regulation of $\mathrm{SC}$ pluripotency, proliferative activity, and differentiation [17-20]. Coordinated control of cellular protein and redox homeostasis by the $\mathrm{UPR}^{\mathrm{ER}}$ and other stress signaling pathways is therefore critical to maintain SC function. Exogenous ER stress likely disrupts this coordination, perturbing regeneration and proliferative homeostasis. Consistent with this model, excessive $\mathrm{UPR}^{\mathrm{ER}}$ activity has been implicated in tumorigenesis $[2,21]$. 


\section{Author Summary}

Loss of proper protein homeostasis (proteostasis) as well as increased production of reactive oxygen species (ROS) is a hallmark of aging. In complex metazoans, these processes can result in proliferative diseases and cancers. The protein folding capacity of the endoplasmic reticulum (ER) is monitored and maintained by the unfolded protein response of the ER $\left(U P R^{E R}\right)$. In this study, we identify a coordinated role of UPR ${ }^{\mathrm{ER}}$ and oxidative stress signaling in regulating the proliferation of intestinal stem cells (ISCs). We find that the ER-stress responsive transcription factor $\mathrm{Xbp} 1$ and the ER-associated degradation pathway component Hrd1 are sufficient and required cell autonomously in ISCs to limit their proliferative activity. This function is dependent on the activities of the stress sensor JNK and the redox-responsive transcription factor $\mathrm{CncC}$, which we have previously identified as regulators of ISC proliferation. We further show here that promoting ER homeostasis in aging ISCs is sufficient to limit age-associated epithelial dysplasia. Our results establish the integration of UPR ${ }^{E R}$ and oxidative stress signaling as a central mechanism promoting regenerative homeostasis in the intestinal epithelium.

To understand the long-term maintenance of epithelial homeostasis in the intestine, detailed insight into the regulation and function of the UPR ${ }^{\mathrm{ER}}$ and its coordination with the redox response in the intestinal epithelium, in a cell-type specific and temporally resolved manner, is required. Here, we have initiated such an analysis, using the Drosophila intestinal epithelium as a model system. The Drosophila ISC lineage exhibits a high degree of functional and morphological similarities with the ISC lineage in the mammalian small intestine [22-24]. When a regenerative response is induced in the intestinal epithelium, ISGs self-renew and give rise to transient, non-dividing progenitor cells called EnteroBlasts (EBs), which differentiate into either absorptive EnteroCytes (ECs) or secretory EnteroEndocrine (EEs) cells, triggered by differential Notch signaling. ISCs are the only dividing cells in the posterior midgut of Drosophila and their entry into a highly proliferative state is regulated by multiple stress and mitogenic signaling pathways, including Jun-N-terminal Kinase (JNK), Jak/Stat, Insulin, Wnt, and EGFR signaling [24,25]. The transcription factor CncC (orthologue of mammalian Nrf2 and worm SKN-1), a master regulator of intracellular redox homeostasis, controls proliferation of ISCs by limiting ROS accumulation [19]. Interestingly, mammalian Nrf2 has been suggested to buffer ROS production during ER stress, while worm SKN-1 has recently been found to coordinate antioxidant gene expression with Xbpl [26,27].

During aging, flies develop epithelial dysplasia in the intestine, caused by excessive ISC proliferation and deficient differentiation of EBs [28,29]. This phenotype is a consequence of an inflammatory condition initiated by dysbiosis of the commensal bacteria, and causes metabolic decline, loss of epithelial barrier function, and increased mortality [30-32]. The ISC-intrinsic mechanisms causing the decline of proliferative homeostasis in the aging intestinal epithelium remain unclear.

Here, we have dissected the role of the $\mathrm{UPR}^{\mathrm{ER}}$ and redox signaling in the control of ISC function and epithelial homeostasis at cellular resolution. We find that ER homeostasis is lost in the aging intestinal epithelium, and that this loss correlates with intestinal dysplasia. Activation of the $\mathrm{UPR}^{\mathrm{ER}}$ within ISCs is required and sufficient for ISC proliferation, and excessive ER stress contributes to the age-associated dysplasia observed in the Drosophila gut. These effects are mediated by changes in the intracellular redox state, which perturb Nrf2/CncC and JNK activities. Accordingly, we find that JNK and Nrf2/CncG act epistatically in the control of ISC proliferation by ER stress. Our findings provide an integrated model for the regulation of ISC activity by redox and proteostatic signaling, and highlight the effects of this integration on epithelial homeostasis.

\section{Results}

\section{$\mathrm{UPR}^{\mathrm{ER}}$ activation in aging Drosophila intestines}

In a recent transcriptome analysis of age-related changes in the Drosophila intestine [32], we noticed that expression of the ER stress-responsive genes $\mathrm{Bip} / \mathrm{Hsc} 70-3 / \mathrm{Hsc3}$, the ER chaperone $G p 93$, and $X b p 1$ are significantly induced in aging guts (Figure 1A). To confirm these findings, we used an antibody against Hsc3 and a reporter for Xbpl expression, Xbpl $1_{\mathrm{p}}:$ dsRed [33], and assessed their expression in young and old guts (Figure 1C, 1D, S1H). Consistent with the RNAseq results, Hsc3 immunoreactivity and Xbpl expression increased throughout the posterior midgut of aging flies (Figure 1C, 1D, S1H), suggesting that the $\mathrm{UPR}^{\mathrm{ER}}$ is activated in the aging intestinal epithelium. Since ER stress has been implicated in deregulation of mammalian ISC function [2,16], and since Drosophila ISGs over-proliferate in aging guts, causing epithelial dysplasia [28,29,32], we assessed whether the UPR ${ }^{\mathrm{ER}}$ is activated in aging ISCs. ISCs and EBs can be identified in the posterior midgut of flies by expression of GFP driven by the esg::Gal4 driver, and ISCs can further be identified by expression of the Notch ligand Delta (DI). In young animals, Hsc3 was expressed at lower levels in progenitor cells than in differentiated cells. In old guts however, Hsc3 expression was strongly increased in progenitor cells, suggesting a specific activation of the UPR ${ }^{\mathrm{ER}}$ in these cells (Figure 1E, Figure $\mathrm{S} 1 \mathrm{H}$ ). We confirmed this using an Xbpl splicing reporter [11,34], which assesses activation of Irel. When this reporter was expressed in ISCs and EBs using esg::Gal4, no activity was detected in young flies, but GFP fluorescence was readily detectable in old guts (Figure 1F).

\section{Control of ISC proliferation by the UPR ${ }^{\mathrm{ER}}$}

To test whether the loss of ER homeostasis is a cause or a consequence of the age-associated over-proliferation of ISCs, we examined the proliferative activity of ISCs in which Xbpl had been knocked down by RNAi (the effectiveness of the RNAi and over-expression constructs used here and below were validated by RT-PCR; Figure S2D). We used the esg::Gal4 driver in combination with tub::Gal80ts, which allows temperature-inducible expression of UAS-controlled dsRNAs in ISCs and EBs (this combination is labeled $\mathrm{esg}^{\mathrm{ts}}$ throughout). Perturbing Xbpl was sufficient to strongly induce ISC proliferation, as measured by the frequency of cells positive for the mitotic marker phospho-Histone $\mathrm{H} 3$ (pH3; Figure 2A), and by the expansion of Dl+/esg+ cells within the epithelium (Figure 2B).

We confirmed the induction of ISC proliferative activity in these conditions by assessing the rate of tissue turnover in flies in which $\mathrm{Xbpl}^{\mathrm{RNAi}}$ and GFP were heritably expressed in ISCs and their daughter cells in response to an ISC-specific recombination event (using esg::Gal4, tubGal80ts, UAS::GFP, UASFlp, act $>$ STOP $>$ Gal4, also termed $\operatorname{esg}^{\text {ts }} \mathrm{F} / \mathrm{O}$, [35]). Knocking down Xbpl greatly accelerated epithelial renewal compared to wild type conditions, further highlighting a role for ER stress in promoting ISC proliferation (Figure S1A). The over-proliferation induced by knocking down Xbpl in ISCs also increased phosphorylation of 
A

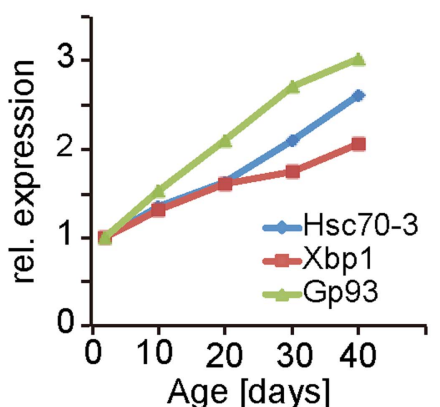

$\begin{array}{llllll}\text { Gene } & 2 d & 10 d & 20 d & 30 d & 40 d \\ & & & & & \\ \text { Gp93 } & 132.5 & 202.9 & 277.7 & 359.7 & 402.4 \\ \text { Hsc70-3 } & 404.5 & 548.2 & 658.6 & 853.0 & 1058.3 \\ \text { Xbp1 } & 448.8 & 586.2 & 721.9 & 781.1 & 927.8\end{array}$

C esg::G4; UAS::nlsGFP/+

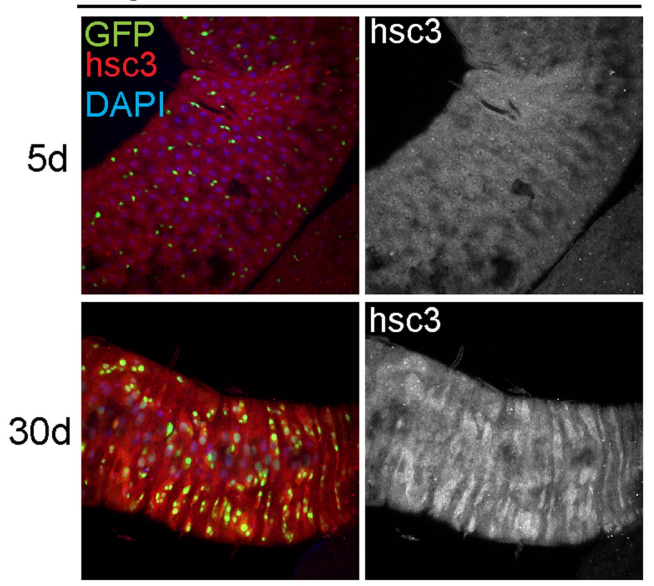

E esg::Gal4,UAS::GFP/+

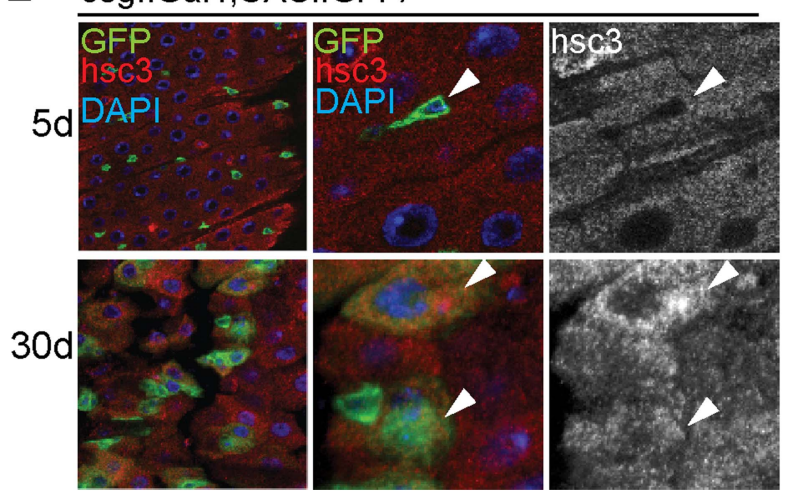

B

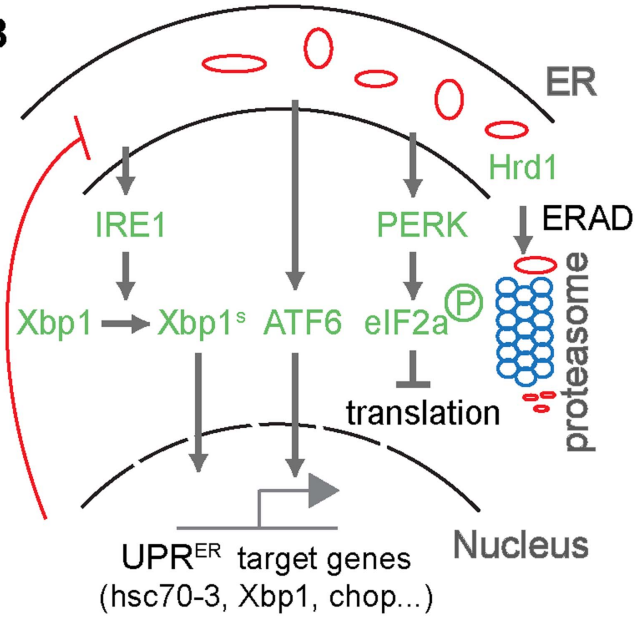

D esg::G4, UAS::GFP, Xbp1P>DsRed

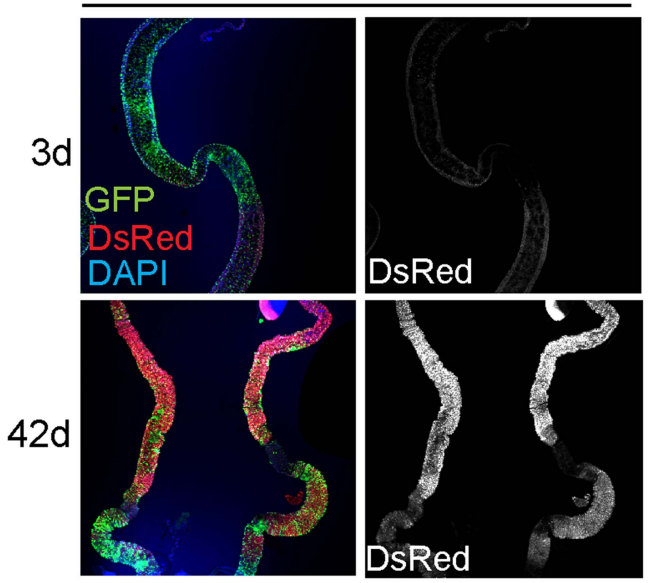

F esg::G4/UAS::Xbp1-EGFP (HG)

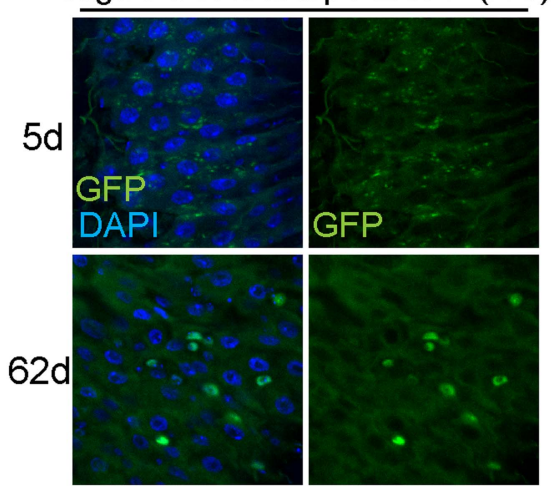

Figure 1. The UPR ${ }^{\mathbf{E R}}$ is activated in aging intestines. (A) Expression of Gp93, Hsc70-3 and Xbp1 in guts from flies of different ages. Expression was determined using RNAseq in experiments described in [32]. Expression values are shown normalized to the $2 \mathrm{~d}$ timepoint in the graph. Raw RPKM values are shown in the table. (B) Major branches of UPR ${ }^{E R}$ signaling pathways in Drosophila. (C) Young (5 day old) and old (30 day old) guts of esg::Gal4, UAS::GFP flies immunostained with anti-Hsc3 antibody (DNA: DAPI, blue; ISCs/EBs: GFP, green; Hsc3, red). The Hsc3 channel is shown separately on the right. (D) Reporter line Xbp $1 p:: D s$ Red visualizes expression of Xbp1 in young (3 day) and old (42 day) guts (DNA: DAPI, blue; ISCs/EBs: GFP, green; DsRed: red). The DsRed channel is shown separately on the right. (E) Enlarged images for intestines of young ( 5 day) and old (30 day) flies (esg::Gal4, UAS:: GFP/+) immunostained with anti-Hsc3 antibody (DNA: DAPI, blue; ISCs/EBs: GFP, green; Hsc3, red). White arrowheads indicate ISCs/ EBs. The Hsc3 channel is shown separately on the right. (F) Xbp1 splicing reporter (UAS::Xbp1-EGFP) expressed in ISCs/EBs using esg::Gal4. Expression of GFP in young ( 5 day) and old (62 day) guts is shown. (DNA: DAPI, blue; GFP, green). The GFP channel is shown separately on the right. See also Figure S1.

doi:10.1371/journal.pgen.1004568.g001 

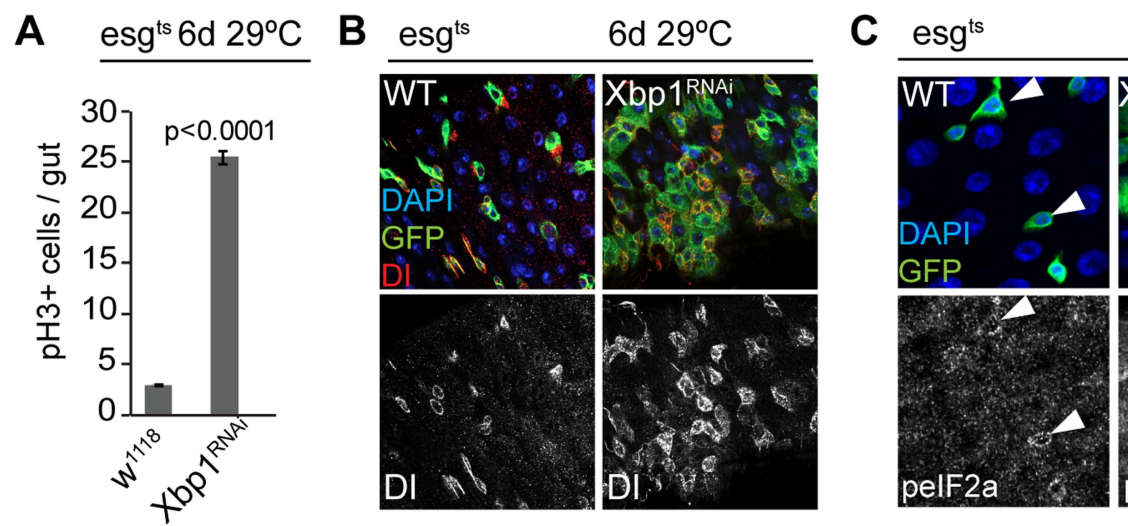

$6 \mathrm{~d} 29^{\circ} \mathrm{C}$
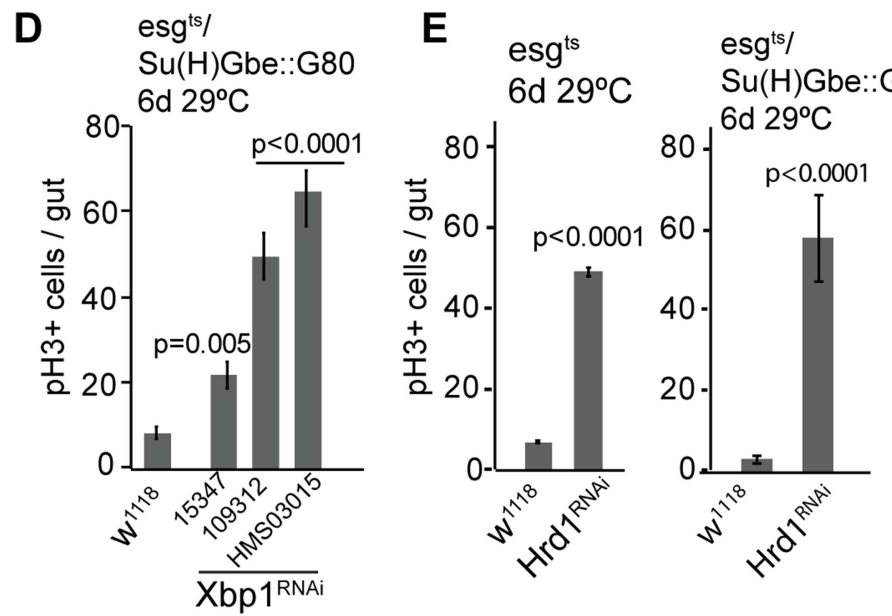

\section{$\mathbf{F}$}

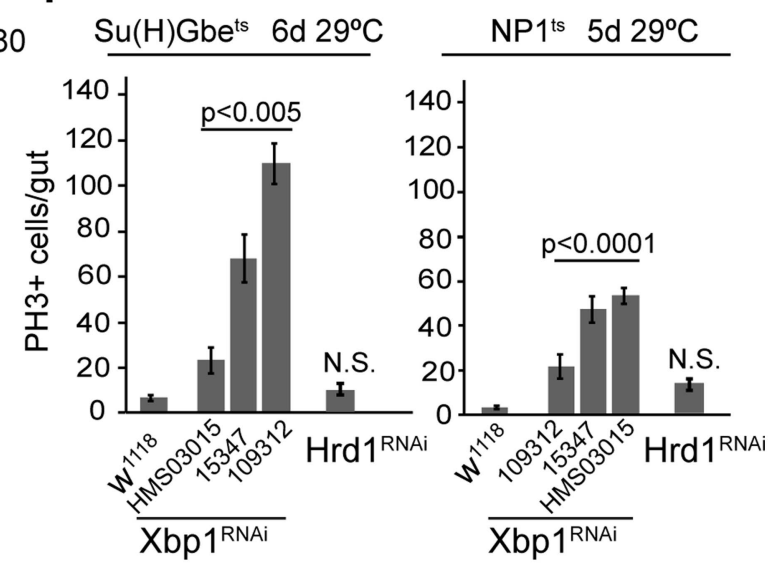

G

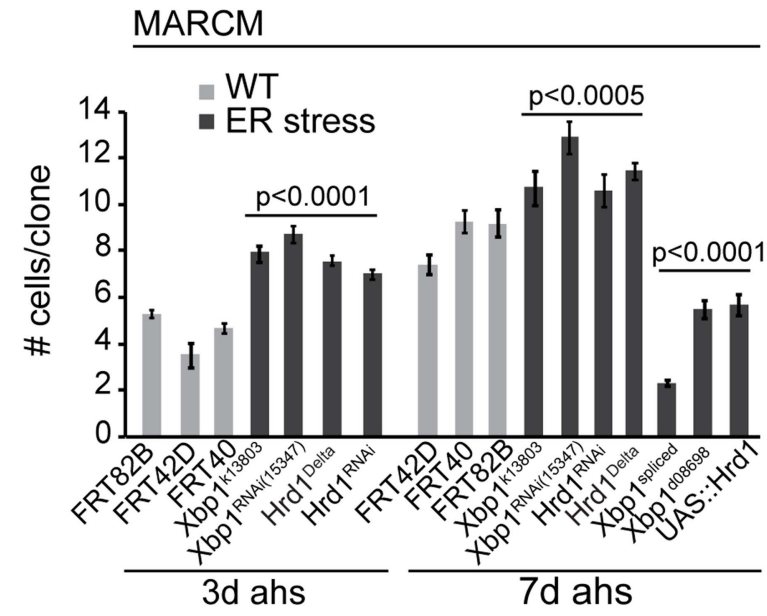

H

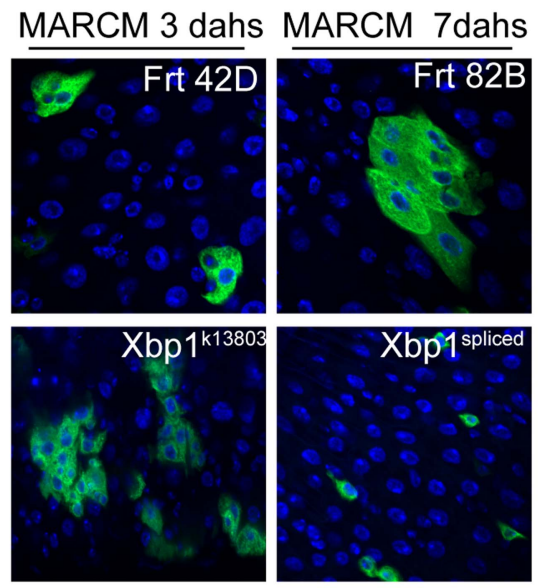

Figure 2. The UPR ${ }^{\mathrm{ER}}$ is sufficient and required in ISCs to promote proliferation. (A) Knockdown of Xbp1 (using Xbp1RNAi ${ }^{15347}$ ) in ISCs/EBs (using esg::Gal4, tubG80 ${ }^{\text {ts }}$ ) leads to ISC over-proliferation in intestines, quantified by counting the number of pH3+ cells/gut. (B) Representative images of wild-type flies and flies with ISC/EB-specific knockdown of Xbp1 (DNA: DAPI, blue; ISCs/EBs marker: GFP, green; ISC marker: DI, red/white). $\mathrm{DI}$ channel is shown separately in lower panels. (C) Loss of Xbp1 or Hrd1 in ISCs/EBs promotes elF2 $\alpha$ phosphorylation in ISCs/EBs, Compared to wildtype control. pelF $2 \alpha$ channel is shown in white in lower panels. Arrowheads for orientation. DNA: DAPI, blue; ISCs/EBs: GFP, green; pelF2 $\alpha$, grey. (D) ISC-specific knockdown of Xbp1 (using esg::Gal4, Su(H)-Gbe::G80,tub::Gal80ts) induces ISC proliferation. 3 different fly lines expressing dsRNA against Xbp1 in a Gal4-sensitive manner (Xbp1RNAi ${ }^{15347}$, Xbp1RNAi ${ }^{109312}$, and Xbp1RNAi ${ }^{H M S 03015}$ ) were used. Averages and SEM are shown. P values from Student's T test, $\mathrm{N}>20$. (E) Knockdown of Hrd1 in ISCs/EBs (using esg::Gal4, tubG80ts), or specifically in ISCs (esgts/Su(H)Gbe::G80) induces ISC proliferation. Averages and SEM are shown. P values from Student's T test, $\mathrm{N}>10$. (F) Quantification of $\mathrm{pH}^{+}$cells in wild-type flies and in flies expressing RNAi constructs against Xbp1 or Hrd1 in EBs (using Su(H)Gbe::Gal4, tub::Gal80ts) or ECs (using NP1::Gal4, tub::Gal80ts). Averages and SEM are shown. $P$ values from Student's $T$ test, $N=10$. (G) Quantification of MARCM clone sizes at 3 days and 7 days after heat shock for Xbp1 and hrd 1 loss-of-function (Xbp1 $1^{\mathrm{k} 13803}, \mathrm{Xbp}^{\mathrm{RNAi}}, \mathrm{Hrd} 1^{\text {Delta }}, \mathrm{Hrd1}^{\mathrm{RNAi}}$ ) or gain-of-function (Xbp1 ${ }^{\text {spliced }}, \mathrm{Xbp}^{\mathrm{d}}{ }^{\mathrm{d} 8698}$ and UAS::Hrd1) conditions. Averages and SEM are shown. $P$ values from Student's T test. Number of clones examined: $n=447$ (3d FRT82B); $n=42$ (3d FRT42D); $n=165$ (3d FRT40); $n=95$ (3d Xbp1 ${ }^{\mathrm{k} 13803}$ ); $\mathrm{n}=268$ (3d Xbp1RNAi); $\mathrm{n}=515$ (3d Hrd1 ${ }^{\text {Delta }}$ ); $\mathrm{n}=262$ (3d Hrd1RNAi); $\mathrm{n}=178$ (7d FRT42D); $\mathrm{n}=215$ (7d FRT40); $\mathrm{n}=394$ (7d FRT82B); 
$\mathrm{n}=123\left(7 \mathrm{~d} \mathrm{Xbp1}{ }^{\mathrm{k13803}}\right) ; \mathrm{n}=270\left(7 \mathrm{~d}\right.$ Xbp1RNAi); $\mathrm{n}=424\left(7 \mathrm{dHrd1} 1^{\text {Delta }}\right) ; \mathrm{n}=119\left(7 \mathrm{~d}\right.$ Hrd1RNAi); $\mathrm{n}=99\left(\right.$ Xbp1 $\left.^{\text {spliced }}\right) ; \mathrm{n}=138\left(\right.$ Xbp1 $\left.^{\text {d08698 }}\right) ; \mathrm{n}=162$ (UAS::Hrd1). (H) Representative images for Xbp1 loss-of-function at 3 days after heat shock and spliced Xbp1 at 7 days after heat shock are shown on the right. (DAPI, blue; GFP, green). See also Figure S1.

doi:10.1371/journal.pgen.1004568.g002

eIF2 $\alpha$, which is phosphorylated in response to ER stress by PERK, indicating that this induction of ISC proliferation is associated with an activation of the $\mathrm{UPR}^{\mathrm{ER}}$ (Figure $2 \mathrm{C}$ ).

We asked whether the proliferative response of ISCs in Xbpl loss of function conditions was induced by changes in ER homeostasis in ISCs specifically, or whether induction of ER stress in EBs or daughter cells was driving ISC proliferation nonautonomously. To address this question, we first restricted expression of $\mathrm{Xbpl}^{\mathrm{RNAi}}$ to ISCs, by combining esg::Gal4 with a transgenic construct that inhibits Gal4 activity in $\mathrm{EBs}(\mathrm{Su}(\mathrm{H})$ Gbe::Gal80; $\mathrm{Su}(\mathrm{H})$-Gbe promoter elements are activated specifically in EBs in response to $\mathrm{Dl} / \mathrm{N}$ signaling in EBs [36]; Figure S2E). Using 3 different dsRNA constructs against Xbpl, we confirmed that Xbpl knockdown specifically in ISCs is sufficient to induce ISC proliferation (Figure 2D, Figure S1G). Xbpl has a complex role in ER homeostasis, serving both as a sensor for ER stress and as a promoter of ER growth and proteostasis [11,34,37], and may also act independently of the ER stress response to regulate ISC proliferation. We therefore tested if independently activating the $\mathrm{UPR}^{\mathrm{ER}}$, by impairing the removal of unfolded proteins in the ER directly, is sufficient to induce ISC proliferation. To this end, we perturbed the ER-associated degradation (ERAD) pathway by knocking down the ERAD-associated E3 ubiquitin ligase $\mathrm{Hrdl}$, which is required for ubiquitination and degradation of unfolded proteins in the ER [38]. Knockdown of Hrd 1 also induced eIF2 $\alpha$ phosphorylation in ISCs (Figure 2G) and increased ISC proliferation, both when driven by $\mathrm{esg}^{\text {ts }}$ and when driven by $\mathrm{esg}^{\text {ts }}$ in combination with $\mathrm{Su}(\mathrm{H}):$ :Gal80 (Figure 2E, Figure S1G). We also examined whether knocking down Xbpl or Hrdl in other cell types of the gut epithelium is sufficient to promote ISC proliferation. Knocking down Xbp1, but not Hrdl in $\mathrm{EBs}$ (using $\mathrm{Su}(\mathrm{H}) \mathrm{Gbe}:$ Gal4 [39] in combination with tub::Gal80ts) or in EGs (using NP1::Gal4, tub::Gal80ts) increased ISC proliferation (Figure 2F), suggesting the existence of an Xbpl-specific non-autonomous effect on ISC proliferation in this tissue. Taken together, our results indicate that loss of ER homeostasis within ISCs induces ISC proliferation. The nonautonomous feedback of Xbpl perturbation in ISC daughter cells on ISG proliferative activity is interesting and will be explored mechanistically in a separate study (Wang et al., in preparation).

If activation of the $\mathrm{UPR}^{\mathrm{ER}}$ is required for the regenerative response of ISCs, perturbation of $\mathrm{UPR}^{\mathrm{ER}}$ components should influence the growth of ISC-derived cell clones. To test this idea, and to determine the requirement for $\mathrm{UPR}^{\mathrm{ER}}$ components in the regulation of ISC activity in homeostatic conditions, we performed linage tracing of mutant stem cells via the MARCM system [40]. Clones generated by ISCs homozygous for the Xbpl loss-offunction allele $X b p 1^{k 13803}$, the Hrdl loss of function allele $h r d 1^{\text {Delta }}$ (a deletion that deletes Hrd1 and CG2126, see methods), or clones expressing $\mathrm{Xbpl}^{\mathrm{RNAi}}$ or $\mathrm{Hrdl}^{\mathrm{RNAi}}$ grew significantly faster than wild-type controls (Figure 2G, Figure 2H, Figure S1BE). Accordingly, clones derived from ISCs over-expressing endogenous Xbpl (using $\mathrm{Xbpl}^{\mathrm{d} 08698}$, a line in which $\mathrm{Xbpl}$ transcription is induced downstream of a transgenic UAS [37,41]), a transgene encoding a constitutively active, spliced version of Xbpl [11], or transgenic Hrdl [42], grew significantly slower than clones derived from wild-type ISGs (Figure 2G, Figure 2H, Figure $\mathrm{S} 1 \mathrm{~F})$. While maintaining ER homeostasis through the $\mathrm{UPR}^{\mathrm{ER}}$ is thus essential to limit ISC proliferation and prevent dysplasia, a functional $\mathrm{UPR}^{\mathrm{ER}}$ is also required for normal homeostatic regeneration.

\section{$\mathrm{UPR}^{\mathrm{ER}}$ as a rheostat for stem cell proliferation}

To further confirm that promoting ER homeostasis within ISCs selectively limits their proliferation, we assessed if increasing the expression of UPR ${ }^{\mathrm{ER}}$ components in ISCs or their daughter cells was sufficient to allay tunicamycin-induced ISC proliferation. Tunicamycin, potently induces ER stress by inhibiting N-linked protein glycosylation and thus impairing protein folding [43]. Feeding tunicamycin very robustly induced ISC proliferation, supporting a role for activation of the $\mathrm{UPR}^{\mathrm{ER}}$ in promoting ISC proliferation (Figure 3A, Figure 3B, Figure 3D, and Figure 3E). Increasing ER stress tolerance by over-expressing endogenous Xbpl, spliced Xbpl, Hrd1, or Hsc3/Bip in ISCs and EBs (using esg::Gal4 and esg $^{\text {ts }}$; spliced Xbpl was expressed only in adults using es ${ }^{\text {ts }}$ ) is sufficient to significantly reduce tunicamycin-induced ISC proliferation (Figure 3A, Figure 3B, Figure 3D, note that expressing spliced Xbpl, endogenous Xbpl (using Xbpl ${ }^{\mathrm{d} 08698}$ or $\left.\mathrm{Xbp1}^{\mathrm{EP} 2112}[37,41]\right)$, as well as Hsc3/Bip also inhibited proliferation induced by oxidative stress inducer paraquat, Figure $3 \mathrm{C}$, Figure 3D,). This inhibition was also observed when spliced Xbpl was over-expressed selectively only in ISCs (using esg $^{\text {ts }}$; $\mathrm{Su}(\mathrm{H})$ Gbe:: Gal80; Figure 3E), but not when spliced Xbpl or Hrdl were expressed in ECs or EBs only (using the EC-specific NP1::Gal4 or the EB-specific $\mathrm{Su}(\mathrm{H}) \mathrm{Gbe}:$ Gal4, both rendered heat-inducible by combination with tub::Gal80ts; Figure 3F, Figure 3G). Altogether, our data indicate that maintaining ER homeostasis in ISCs is critical for long-term ISC quiescence, while an active $\mathrm{UPR}^{\mathrm{ER}}$ within ISCs is required and sufficient for ISC proliferation under homeostatic conditions, as well as in response to ER or oxidative stress. To assess whether engaging the $\mathrm{UPR}^{\mathrm{ER}}$ is universally required for ISC proliferation, we assessed if reducing ER stress by over-expressing spliced Xbpl was sufficient to limit ISC proliferation in a range of mitogenic conditions. ISC proliferation can be triggered through the JNK or the insulin/IGF signaling (IIS) pathways by over-expressing the JNK Kinase Hemipterous (Hep) [28] or the Insulin Receptor (InR) [44-46]. Over-expression of spliced Xbpl was sufficient to inhibit ISC proliferation in both conditions (Figure S2A, Figure S2B; this inhibition is not due to apoptosis of ISCs, as ISCs were readily observed even at 14 days after inducing expression of Hep and/or Xbpl $1^{\text {spliced}}$ ).

Modulating Xbpl activity further influenced the growth of ISC/EE tumors that accumulate due to defective EB differentiation in Notch loss of function conditions: While spliced Xbpl prevented tumor formation, loss of Xbpl exacerbated the growth of these tumors (Figure S2C). By regulating ER homeostasis, Xbpl thus serves as a rheostat broadly controlling ISC proliferative activity.

\section{ROS-mediated control of ISC proliferation by the UPR ${ }^{\mathrm{ER}}$}

The control of ISC proliferation by the UPR ${ }^{\mathrm{ER}}$ resembles ISC control by ROS, which can trigger dysplastic over-proliferation of ISGs, but are required for proliferation during homeostatic regeneration [19,24]. Oxidative stress and ER stress are tightly linked: perturbation of redox homeostasis results in the accumulation of misfolded proteins and activation of the $\mathrm{UPR}^{\mathrm{ER}}$, and ER stress results in cytosolic oxidative stress [47-49]. To explore the 
A

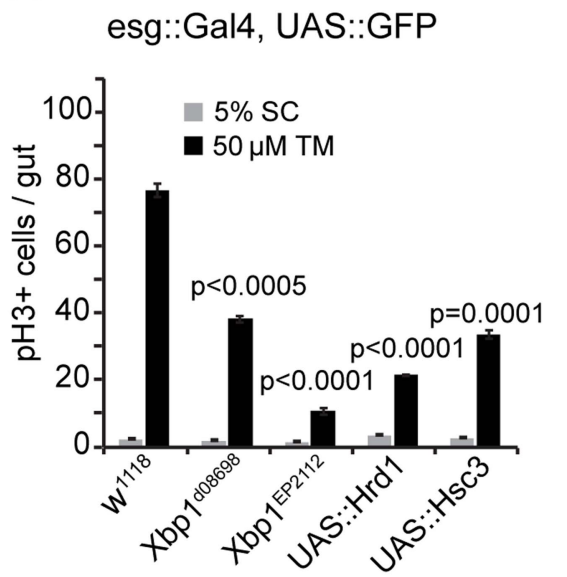

B esg::Gal4, UAS::GFP; tubG80ts>

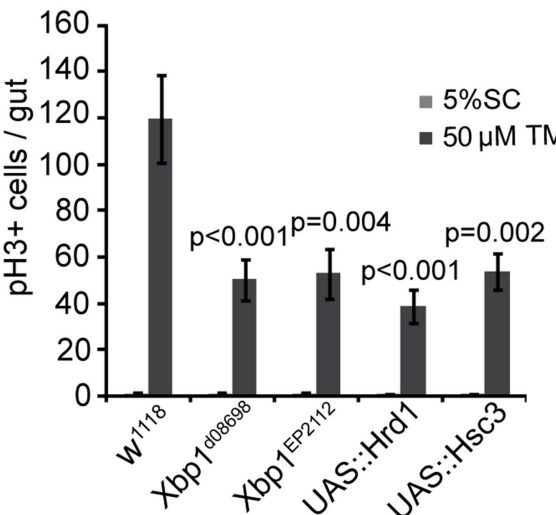

D

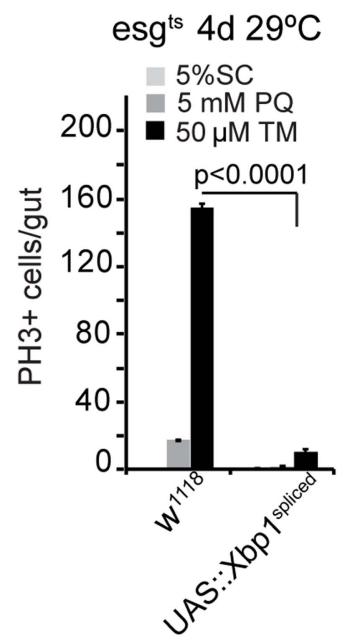

E

\section{esg ${ }^{\text {ts }} /$ \\ esg 1 is} $4 \mathrm{~d} 29^{\circ} \mathrm{C}$

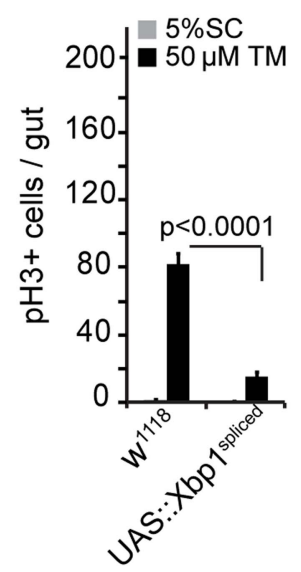

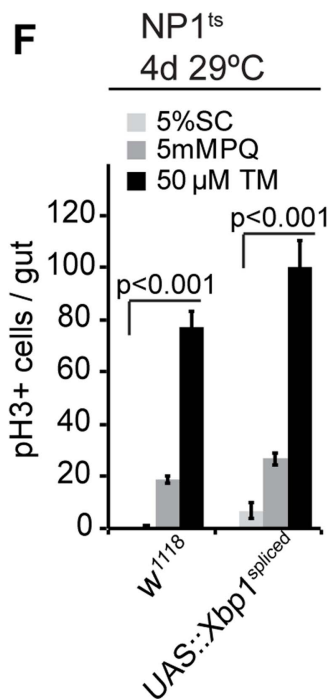

C esg::Gal4, UAS::GFP; tubG80ts>
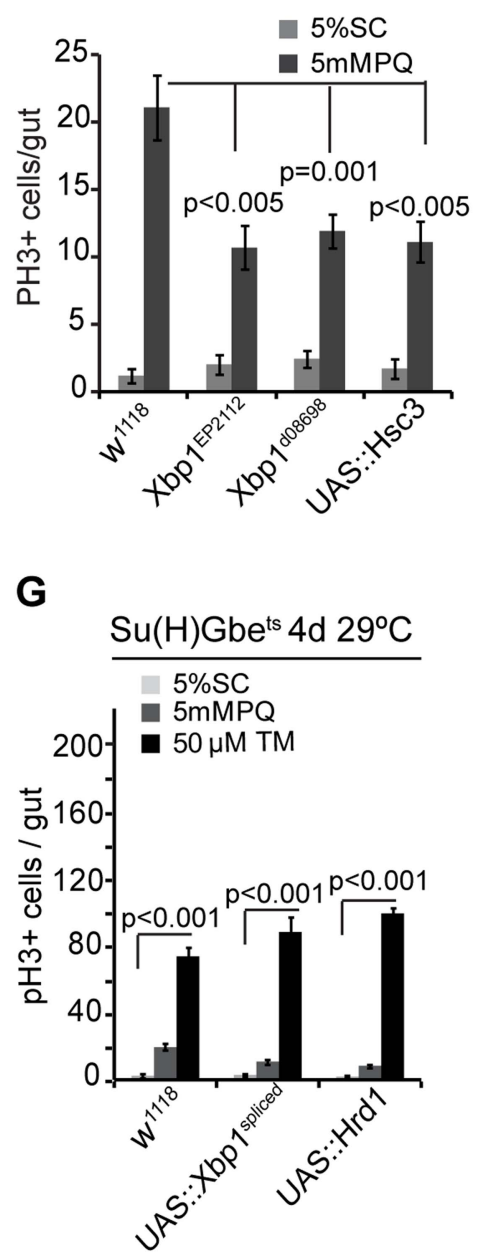

Figure 3. The UPR ${ }^{E R}$ as a rheostat for stem cell proliferation. (A) Quantification of $\mathrm{pH}^{+}$cells in wild-type flies and in flies over-expressing Xbp1 $\left(x b p 1^{d 08698}, x b p 1^{E P 2112}\right)$, Hrd1, or Hsc3 under the control of esg::Gal4 exposed to mock treatment (5\% sucrose) or tunicamycin (TM). Averages and SEM are shown. $P$ values from Student's $T$ test, $N=10$. (B) Quantification of $p H 3^{+}$cells in wild-type flies and in flies over-expressing $X b p 1$ $\left(X b p 1^{d 08698}, X b p 1^{E P 2112}\right)$, Hrd1, or Hsc3 under the control of esg::Gal4, UAS::GFP; tub::Gal80 ${ }^{\text {ts }}$ exposed to mock treatment (5\% sucrose) or tunicamycin (TM). Averages and SEM are shown. P values from Student's T test, $\mathrm{N}=10$. (C) Quantification of $\mathrm{pH}^{+}$cells in wild-type flies and in flies over-expressing $\mathrm{Xbp} 1\left(\mathrm{Xbp} 1^{\text {dos698 }}, \mathrm{Xbp}^{\mathrm{EP} 2112}\right)$, or Hsc3 under the control of esg::Gal4, UAS-GFP; tubG80 ${ }^{\text {ts }}$ exposed to mock (5\% sucrose) and oxidative-stress inducer paraquat (5 mM PQ). Averages and SEM are shown. P values from Student's Test, $N=10$. (D) Spliced Xbp1 prevents ISC over-proliferation induced by excessive ER stress. Quantification of mitotic figures of wild-type flies and flies over-expressing spliced Xbp1 (Xbp1 ${ }^{\text {spliced }}$ ) in ISCs/EBs (esg::Gal4, UASGFP; tubG80 ${ }^{\text {ts }}$ ) exposed to mock treatment ( $5 \%$ sucrose) or paraquat $(5 \mathrm{mM})$ or tunicamycin $(50 \mu \mathrm{M})$. Averages and SEM are shown. P values from Student's Test, $N=10$. (E) Over-expressing spliced Xbp1 in ISCs only (using esg::Gal4, Su(H)-Gbe::G80,tub::Gal80ts) inhibits stress-induced ISC proliferation. Averages and SEM are shown. P values from Student's T test, N $>10$. (F) Over-expressing spliced Xbp1 in ECs has no effect on ISC proliferation upon stress. Quantification of $\mathrm{PH}^{+}$cells in intestines of wild-type flies and in flies expressing Xbp $1^{\text {spliced }}$ specifically in ECs (using NP1::Gal4; tub::Gal80 ${ }^{\text {ts }}$ ). Averages and SEM are shown. P values from Student's T test, $\mathrm{N}=10$. (G) Quantification of pH3 ${ }^{+}$cells in intestines of wild-type flies and in flies expressing spliced Xbp1 (Xbp1 ${ }^{\mathrm{DS} \Delta 28}$ ) or Hrd1 specifically in EBs (using Su(H)Gbe::Gal4,tubG80 ${ }^{\text {ts }}$ ) exposed to mock treatment $(5 \%$ sucrose), paraquat $(5 \mathrm{mM})$, or tunicamycin $(50 \mu \mathrm{M})$. Averages and SEM are shown. P values from Student's Test, $\mathrm{N}=10$. See also Figure S1, S2. doi:10.1371/journal.pgen.1004568.g003

relationship between ER stress, oxidative stress, and the UPR ${ }^{\mathrm{ER}}$ in the ISC lineage, we assessed changes in intracellular redox homeostasis in ISCs deficient in Xbpl or Hrdl (Figure 4A). Both conditions resulted in significantly increased fluorescence of dihydro-ethidium (DHE, a redox-sensitive dye that can be used to detect ROS accumulation in live intestines $[17,19])$ compared to wild-type progenitor cells (Figure 4A). Over-expression of spliced Xbpl, in turn, resulted in decreased DHE fluorescence in ISCs even under stress conditions (Figure 4B).

To further dissect the relationship between ER stress and oxidative stress, we perturbed the peroxiredoxin Jafracl, which strongly influences intracellular redox homeostasis and regulates ISC proliferation $[19,50]$. Knockdown of Jafracl was sufficient to increase ISC proliferation, and this increase was insensitive to the expression of spliced Xbpl (Figure 4G). Over-expression of the anti-oxidant enzymes glutathione peroxidase I (GTPx-1) or Catalase (Cat), on the other hand, inhibited tunicamycin-induced ISC proliferation (Figure 4D), while knocking down Jafracl in ISCs prevented the inhibition of tunicamycin-induced ISC proliferation by spliced Xbpl (Figure 4E). Increased ROS production thus acts downstream of $\mathrm{Xbpl}$ in the regulation of ISC proliferation. 


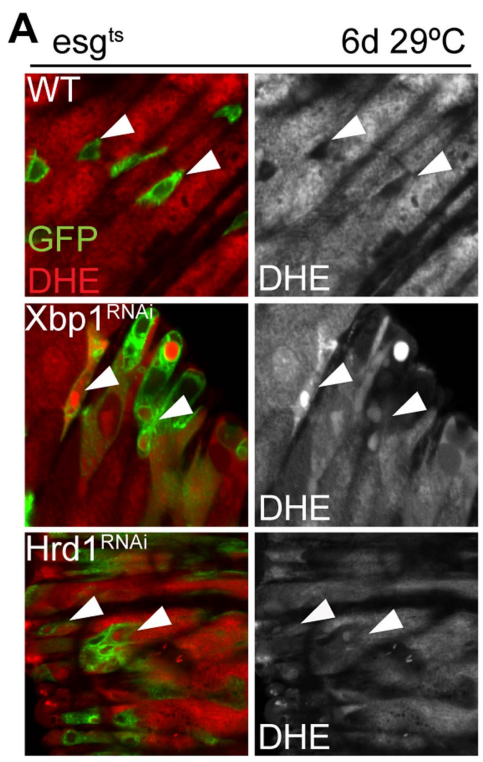

C

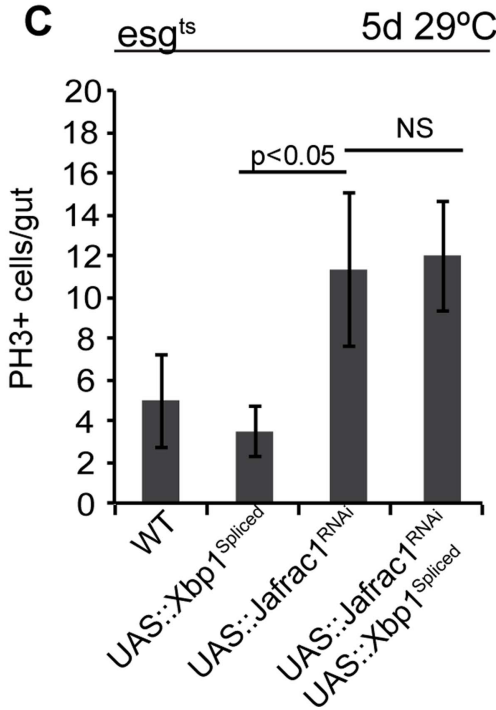

B
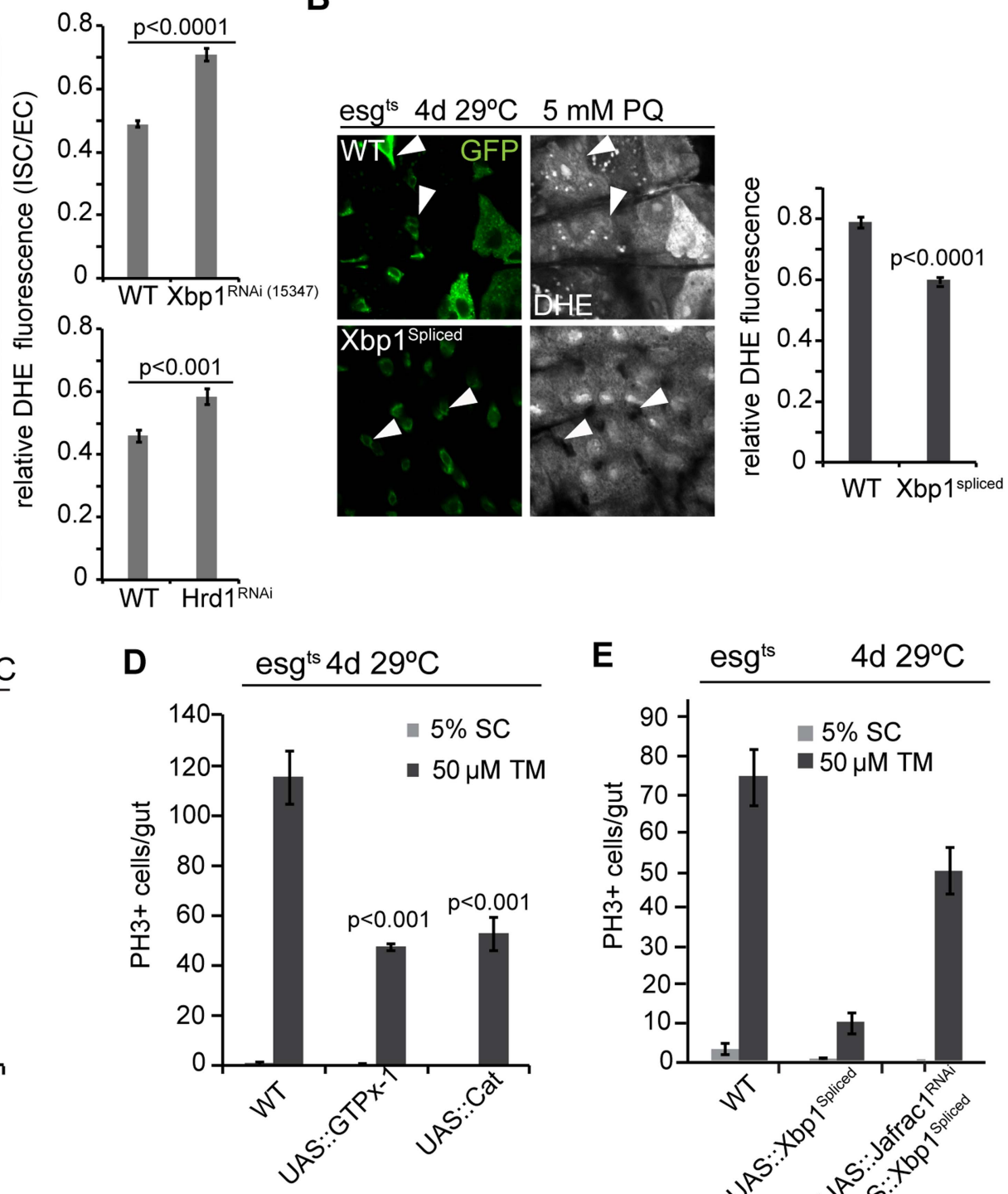

Figure 4. Coordinated control of ISC proliferation by the UPR ${ }^{E R}$ and ROS signaling. (A) Increased ROS in ISCs deficient in Xbp1 or Hrd1. Relative DHE fluorescence intensity is calculated by the ratio of fluorescence intensity in ISCs/EBs and nearby ECs. ISCs/EBs identified by GFP. Arrowheads point to selected ISCs/EBs. GFP, green; DHE red; DHE is shown as separate channel in white. Averages and SEM for relative DHE intensity are shown. P values from Student's T test, N>200 (from 6-7 WT, Xbp $1^{\mathrm{RNAi}}$ guts), N $>100$ (from 3-4 WT, Hrd1 $1^{\mathrm{RNAi}}$ guts). (B) Over-expression of spliced Xbp1 in ISCs/EBs (using esg::Gal4, tubG80 ${ }^{\text {ts }}$ ) resulted in decreased DHE fluorescence in ISCs under Paraquat treatment. Relative DHE fluorescence intensity is quantified for wild-type flies and flies over-expressing spliced Xbp1. Arrowheads point to selected ISCs/EBs. GFP: green. DHE is shown as separate channel in white. Averages and SEM are shown. P values from Student's Test. (C) Frequency of pH3+ cells are quantification for wild-type fly and flies expressing spliced Xbp1 only, Jafrac1 loss-of-function only, and for flies coexpressing spliced Xbp1 and Jafrac1 loss-of-function. Averages and SEM are shown. P values from Student's Test. $N=10$. (D) Over-expression of anti-oxidant enzyme GTPx-1 or Cat in ISCs/EBs (esg::Gal4,tubG80ts) inhibits tunicamycin-induced ISC proliferation. Averages and SEM are shown. P values from Student's T test. N $>10$. (E) Quantification of pH3 ${ }^{+}$cells in wild-type flies, in flies over-expressing spliced Xbp1 only, and in flies expressing both Jafrac1 loss-of-function and spliced Xbp1 under the control of esg::Gal4, UAS-GFP; tubG80 $0^{\text {ts }}$ exposed to mock (5\% sucrose) and tunicamycin. Averages and SEM are shown. P values from Student's Test. $\mathrm{N}=10$. doi:10.1371/journal.pgen.1004568.g004

Increased ISC proliferation in Xbpl or Hrdl loss of function conditions, or in response to tunicamycin treatment, was associated with increased phosphorylation of JNK in Dl+ ISGs (Figure 5A, Figure S3B), and activation of the JNK target gene puckered in all cells of the intestinal epithelium, including ISGs and neighboring EGs (Figure 5B-D, Figure S3A, Figure S3B). This activation can be repressed by over-expressing spliced Xbpl, GTPx-1, or Cat in ISCs, suggesting that JNK is activated in response to ER stress-mediated ROS production (Figure 5D).
Since JNK activation in ISCs promotes their proliferative activity [28], we tested whether JNK activity was required for ISC proliferation in Xbpl loss of function conditions. Indeed, expression of $\mathrm{Bsk}^{\mathrm{RNAi}}$, or of a dominant-negative version of Bsk $\left(\mathrm{Bsk}^{\mathrm{DN}}\right)$, reduced proliferation of ISCs in which Xbpl was knocked down, and in animals exposed to tunicamycin (Figure 5E). These results suggest that activation of JNK in response to ER-stress-induced ROS production is required in ISGs to induce proliferation. 
A

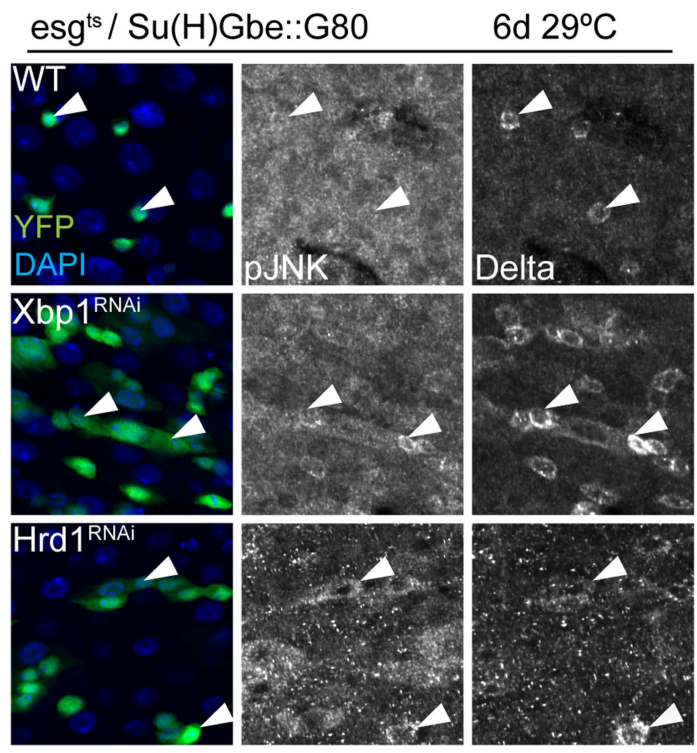

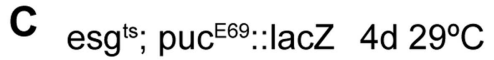

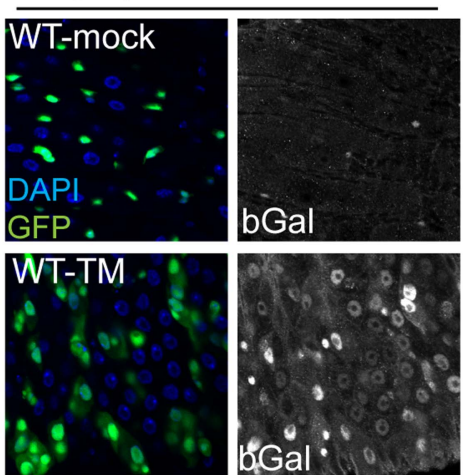

$\mathbf{E}$

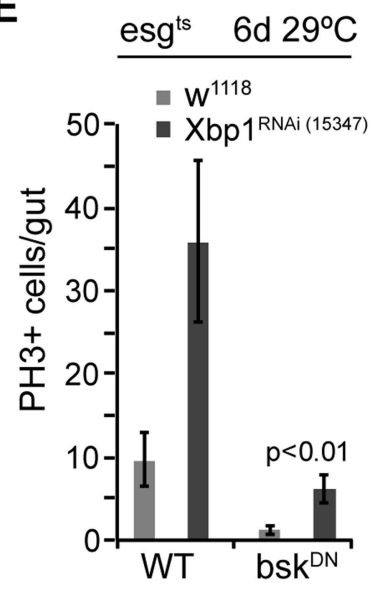

B
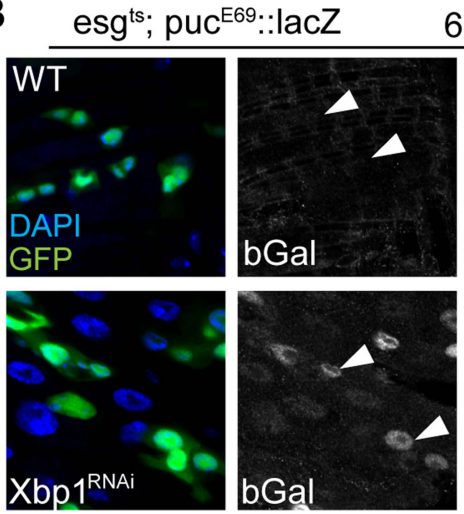

$6 \mathrm{~d} 29^{\circ} \mathrm{C}$
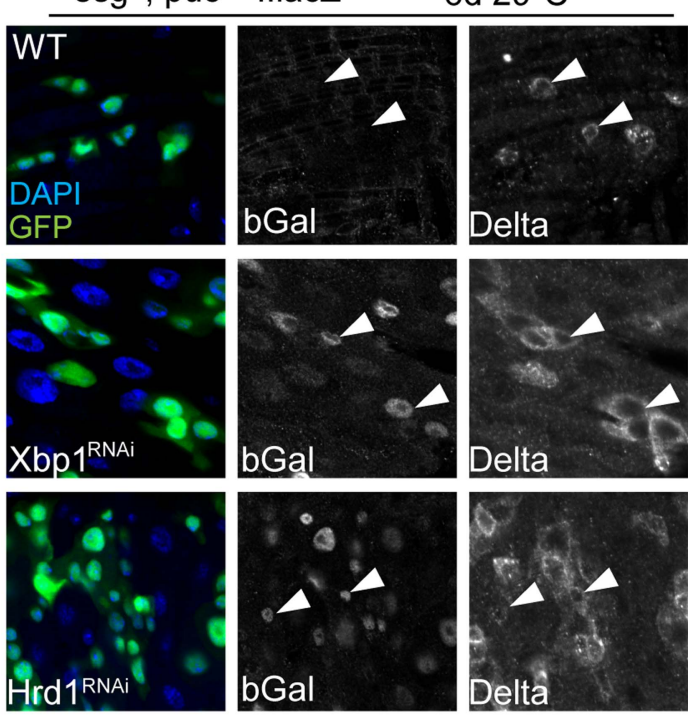

D

$$
\text { esg }^{\text {ts; }} ; \text { puc }^{\mathrm{E} 69 \cdot:: \operatorname{lacZ} \quad 4 \mathrm{~d} 29^{\circ} \mathrm{C}}
$$
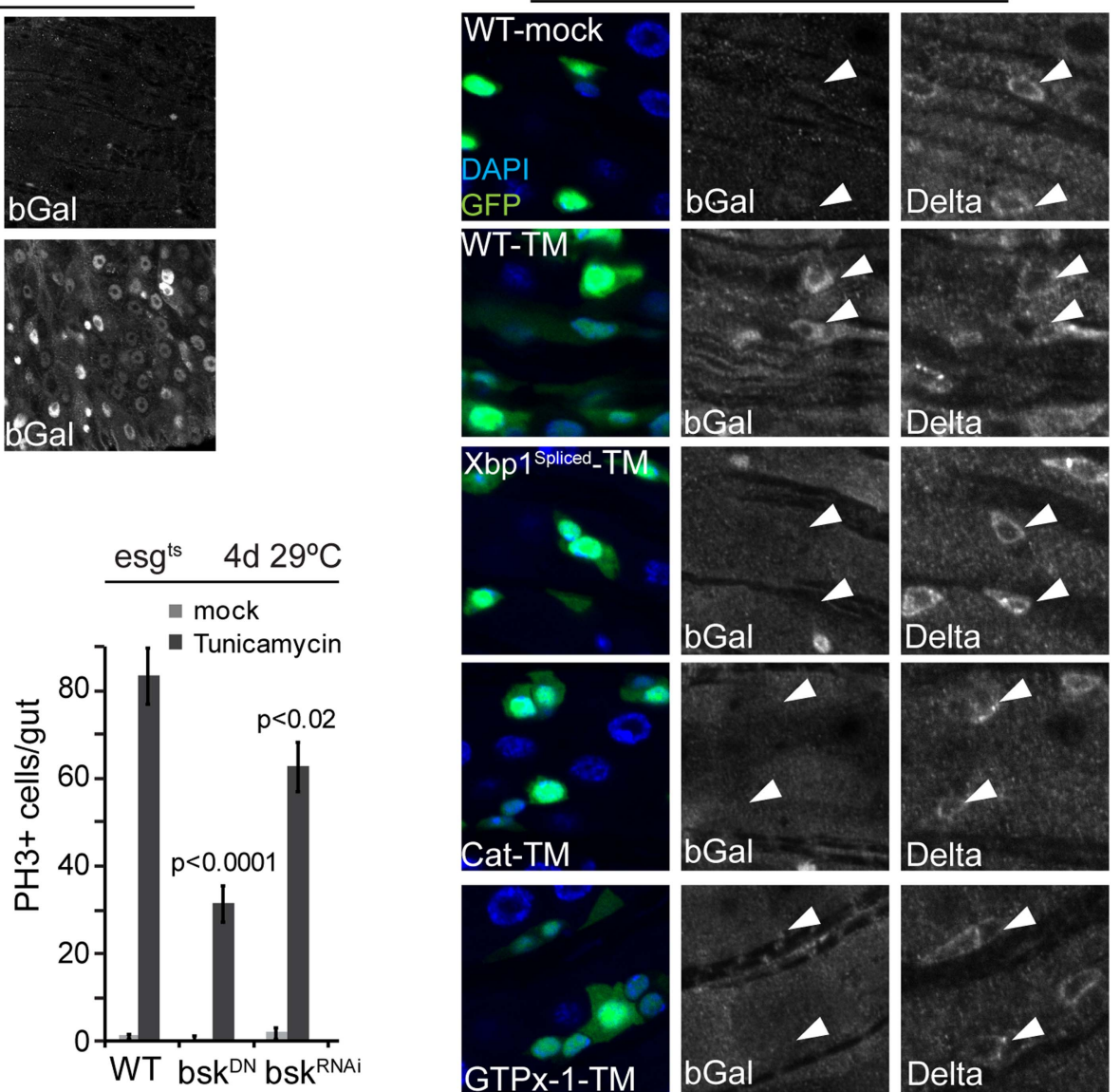

Figure 5. JNK is activated by UPR ${ }^{\text {ER }}$-induced ISC proliferation. (A) JNK is phosphorylated in ISCs when Xbp1 or Hrd1 is knocked down specifically in ISCs. DAPI, blue; GFP, green; pJNK or DI shown as separate channels in white. (B) JNK activation when Xbp1 or Hrd1 is knocked down in ISCs/EBs. Anti bGal antibody staining to detect lacZ expression from the puc ${ }^{\mathrm{E} 69}:$ :lacZ reporter. DAPI, blue; GFP, green; bGal or DI is shown as separate channel in white. (C) JNK activation by tunicamycin exposure. Anti bGal antibody was used to detect expression of lacZ from a puc::IacZ reporter (esg::Gal4, UAS::GFP, tubG80ts/pucE69::IacZ). Flies were exposed to mock treatment (5\% sucrose) or tunicamycin (50 uM in 5\% sucrose). DNA: DAPI, blue; ISCs/EBs: GFP, green; bGal, white. (D) Over-expression of spliced Xbp1 or of GTPx-1 or Cat in ISCs/EBs (esg::Gal4,tubG80ts) represses tunicamycin-induced JNK activation. bGal antibody staining detecting lacZ expression from the puc ${ }^{\mathrm{E}}$ ::IacZ reporter. DNA: DAPI, blue; ISCs/EBs: GFP, green; bGal, white; DI,white). (E) Repressing JNK activity (using Bsk $^{\mathrm{RNAi}}$ or Bsk ${ }^{\mathrm{DN}}$ ) in ISC/EBs (using esg ${ }^{\mathrm{ts}}$ ) inhibits ISC over-proliferation induced by loss of Xbp1 or by tunicamycin treatment. Averages and SEM are shown. P values from Student's T test, $N=10$. See also Figure S3. doi:10.1371/journal.pgen.1004568.g005 


\section{Coordinated control of ISC proliferation by the UPR ${ }^{\mathrm{ER}}$ and the Keap1/CncC pathway}

How do Xbpl and the UPR ${ }^{\mathrm{ER}}$ regulate ISC proliferation? Since promoting ER homeostasis by increasing Xbpl activity or by stimulating the ERAD pathway was sufficient to limit ISC proliferation in all tested stress and mitogenic conditions, and since the Nrf2 homologue CncC exerts a similar effect on ISC proliferation [19], we asked whether CncG activity was influenced by the ER stress response.

To test whether the UPR ${ }^{\mathrm{ER}}$ influences CncG activity in ISCs, we used a gstD1::lacZ construct that responds to $\mathrm{CncC}$ activity in ISCs [19,51]. Strikingly, loss of Xbpl or Hrdl was sufficient to inhibit gstD 1::lacZ expression in ISCs, while ISCs over-expressing spliced Xbpl maintained high gstD1::lacZ expression (Figure 6A). We confirmed the modulation of Xbpl activity in these cells by detecting expression of the Xbpl target hsc3 (Figure 6A). When ER stress was induced by treating animals with tunicamycin, gstD1::lacZ expression was reduced in ISCs, and this inhibition could be alleviated by over-expressing $\mathrm{CncC}$, Xbpl, or $\mathrm{Hrdl}$ (Figure 6B). The same results were obtained when spliced Xbpl was expressed (Figure S4A).

Loss of ER homeostasis thus reduces CncG activity in ISCs, suggesting that $\mathrm{CncC}$ inhibition is a required component of the ER stress response in the regulation of ISC proliferation. To test this idea, we assessed if ISC proliferation is influenced by the interaction between Xbpl and CncC. ISGs mutant for the CncGspecific E3 ubiquitin ligase Keapl do not divide, due to impaired inhibition of CncG activity [19]. ISCs deficient in both Xbpl and Keapl did not divide either, suggesting that CncC acts downstream of $\mathrm{Xbpl}$ in the regulation of ISC proliferation (Figure 6C, Figure 6D). Accordingly, knocking down Keapl or over-expressing CncC was sufficient to rescue ISC over-proliferation caused by loss of Xbpl (Figure 6E, Figure S4B). Overexpressing Cnc $\mathrm{C}$ was also sufficient to inhibit proliferation induced by tunicamycin treatment (Figure 6F). At the same time, loss of CncC was not sufficient to rescue the proliferation defect of ISCs over-expressing spliced Xbpl, suggesting that Xbpl inhibits ISC proliferation not only by preventing CncG inhibition, but by additional mechanisms, most likely by inhibiting PERK activation through ER stress (Figure 6G).

\section{Improved ER quality control in ISCs alleviates age-related intestinal dysplasia}

The age-associated activation of the $\mathrm{UPR}^{\mathrm{ER}}$ in ISCs, and the control of ISC proliferation by the UPR ${ }^{\mathrm{ER}}$, suggested that ER stress in ISCs also plays an important role in promoting agerelated dysplasia. To address this question, we asked whether promoting ER homeostasis in progenitor cells is sufficient to limit dysplasia. Xbpl or Hrdl over-expression was sufficient to maintain expression of gstD 1 ::lacZ, indicating that CncC activity, which declines with age in ISCs [19] was maintained (Figure 7A). Accordingly, Hrdl expression prevented the age-related increase in $h s c 3$ expression in ISGs (Figure S5A; CncG or Keapl ${ }^{\text {RNAi }}$ expression also preserve gstDl::lacZ expression, confirming the responsiveness of the reporter to CncC activity; Figure S5B). As expected, the same genetic conditions also limit ISC proliferation in aging flies, preventing dysplasia (Figure 7B) [19].

\section{Discussion}

Our results establish a critical role for the coordination of oxidative and ER stress responses in the control of stem cell function, proliferative homeostasis and regenerative capacity in the Drosophila intestine. As previously observed for ROS signaling
[19,24], we find that ER stress not only promotes ISC proliferation, but that the $\mathrm{UPR}^{\mathrm{ER}}$ is also required for ISC proliferation under basal, homeostatic conditions. The UPR ${ }^{\mathrm{ER}}$ thus emerges as a rheostat regulating ISC proliferation under both stress and homeostatic conditions. Our results suggest that the tissue-wide increase in ER stress in the aging intestinal epithelium perturbs this regulation, resulting in intestinal dysplasia.

The consequences of perturbing ER homeostasis in the intestinal epithelium are reminiscent of similar effects in Xbpldeficient mice, where loss of Xbpl promotes ISC proliferation and intestinal tumorigenesis [2]. At the same time, a recent study suggests that $\mathrm{UPR}^{\mathrm{ER}}$ components are primarily expressed in transit amplifying cells of the intestinal epithelium, and that activation of the UPR ${ }^{\text {ER }}$ (specifically the PERK branch) promotes differentiation of intestinal epithelial stem cells [16]. The Drosophila midgut epithelium does not contain a transit amplifying cell population, yet our data suggest that a role for the UPR ${ }^{\mathrm{ER}}$ in the control of ISC activity is conserved.

The requirement for $\mathrm{CncC}$ inhibition in $\mathrm{ER}$ stress-mediated activation of ISC proliferation highlights the integrated control of ISC activity by oxidative and ER stress signals. We propose that Xbpl, by promoting ER homeostasis, limits ROS accumulation in ISCs and thus maintains ISC quiescence (Figure 7D, Figure 7E). Excessive ROS results in JNK activation, which in turn activates Fos and inhibits CncC in ISCs, triggering proliferation $([19,52]$ and $\mathrm{Li}$, Hochmuth and Jasper, unpublished results).

This coordination of ER and oxidative stress responses by $\mathrm{CncC}$ and the UPR ${ }^{\mathrm{ER}}$ is likely to be complex. In C. elegans the $\mathrm{UPR}^{\mathrm{ER}}$ coordinates transcriptional regulation of anti-oxidant genes with the CncC homologue SKN-1 [27]. Interestingly, SKN-1 can also directly control the expression of $\mathrm{UPR}^{\mathrm{ER}}$ components (including Xbp1, ATF-6 and Bip) by binding to their promoter regions, independent of oxidative stress [27]. Studies in worms have further established the UPR ${ }^{\mathrm{ER}}$ as a critical determinant of longevity, and Xbpl extends lifespan by improving ER stress resistance [53,54]. Strikingly, local activation of the $\mathrm{UPR}^{\mathrm{ER}}$ can trigger $\mathrm{UPR}^{\mathrm{ER}}$ responses in distant tissues, indicating that endocrine processes exist that coordinate such stress responses across cells and tissues [53-56]. Our results support the notion that improving proteostasis by boosting ER folding capacity improves long-term tissue homeostasis. These effects seem to be largely mediated by cellautonomous integration of the $\mathrm{UPR}^{\mathrm{ER}}$ and redox response by JNK and CncC, but we also observe non-autonomous effects of ER stress on ISC proliferation when knocking down Xbpl in EBs or ECs selectively. Furthermore, JNK is activated broadly in the intestinal epithelium when Xbpl or Hrdl are knocked down in ISCs and EBs, suggesting that non-autonomous interactions between cells experiencing ER stress also influence the regenerative response of this tissue. The molecular events regulating the coordination between cell-autonomous and non-autonomous events in the ER stress response of ISCs are subject of current investigation (Wang et al., in preparation).

In the small intestine of mice, the $\mathrm{UPR}^{\mathrm{ER}}$ influences regenerative activity not only by influencing ISCs and transit amplifying cells directly, but also by influencing intestinal immune homeostasis. Loss of Xbpl in intestinal epithelial cells (IECs) leads to apoptosis of secretory Paneth cells and goblet cells, and this pathology is associated with inflammation and higher risk of IBD $[1,3]$. Deregulation of innate immune responses by the UPR ${ }^{\mathrm{ER}}$ is also found in human patients [1,3,57], as well as in C. elegans $[3,58]$. It can therefore be anticipated that the age-related increase in ER stress in the fly intestine also influences innate immune homeostasis and may contribute to commensal dysbiosis, which we have recently shown to be a driving factor in the age-related loss of 
A

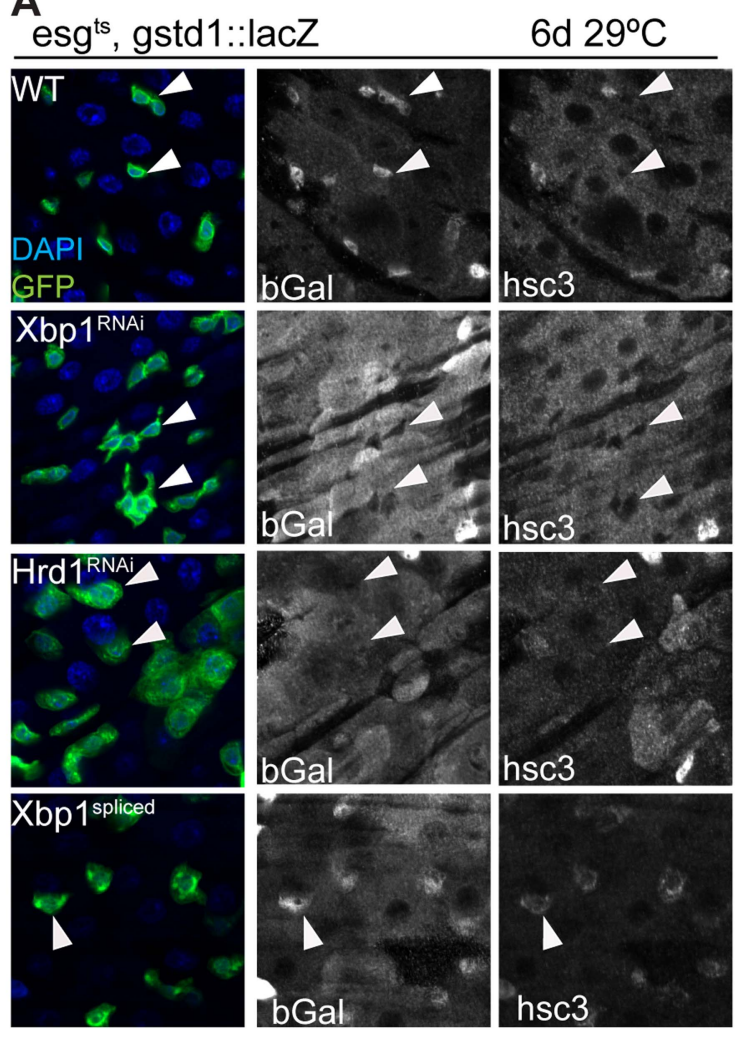

C MARCM 82B 7dahs
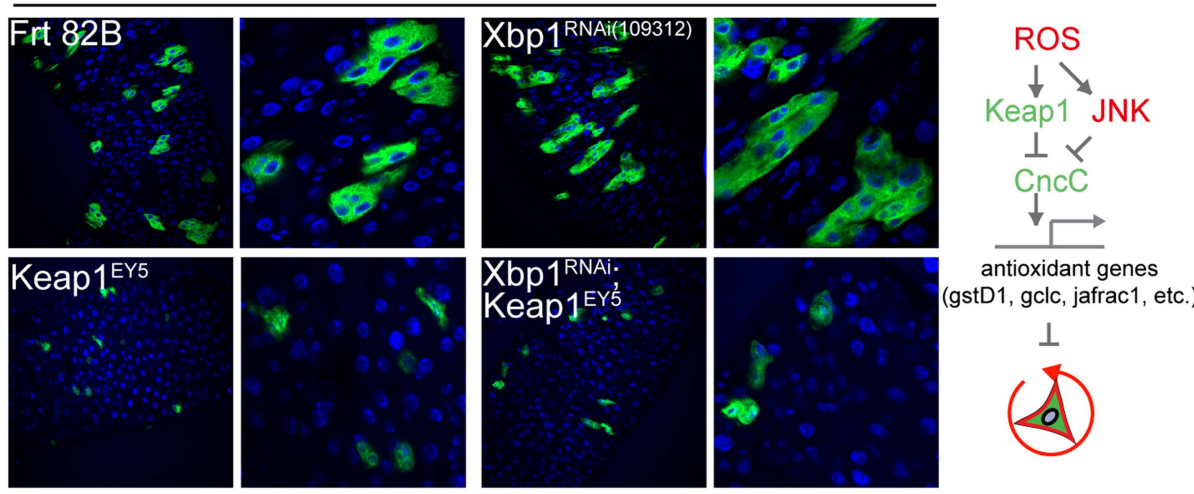

B
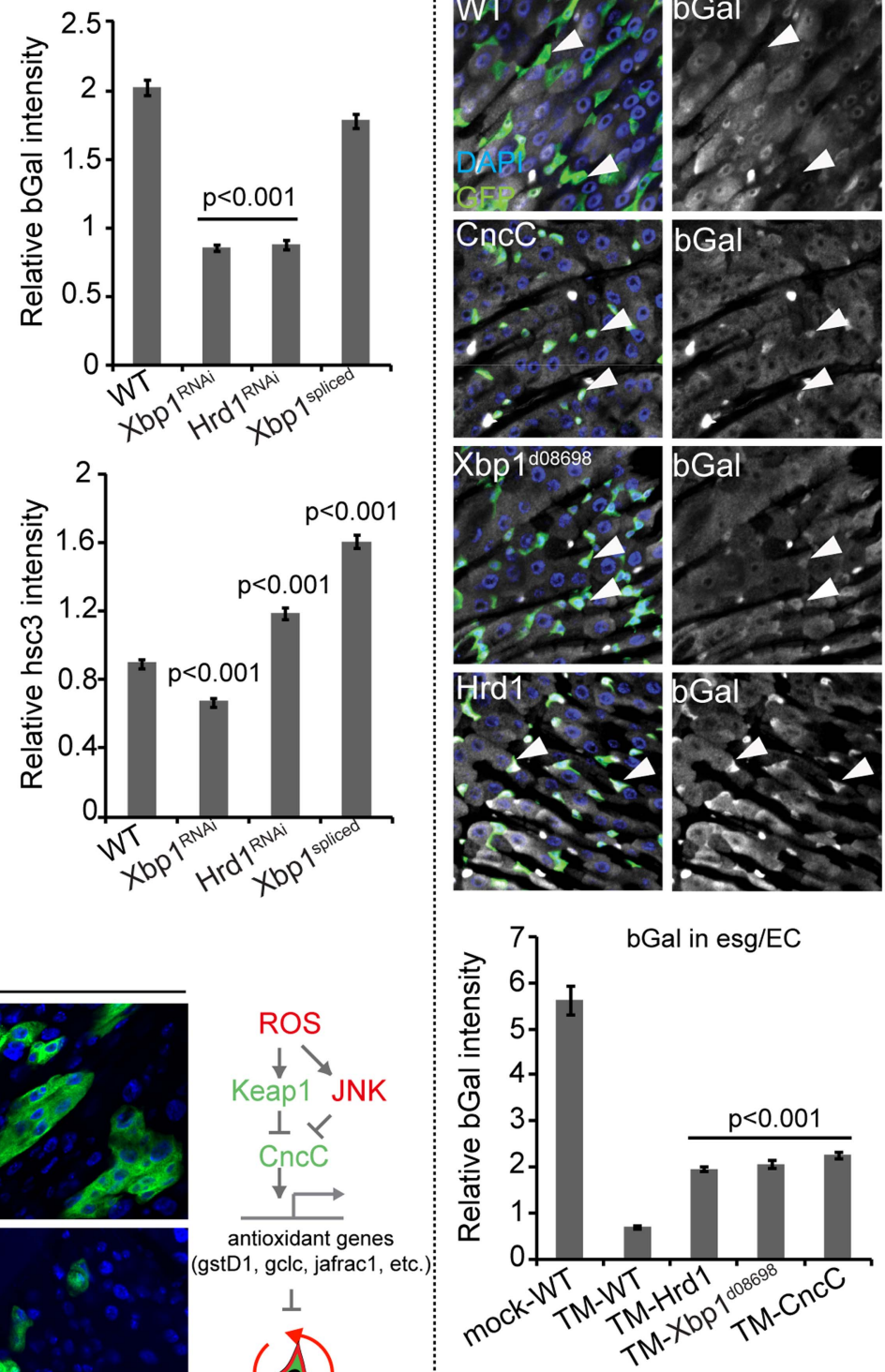

D MARCM82B 7dahs E

E esg $^{\text {ts }} \quad 6 \mathrm{~d} 29^{\circ} \mathrm{C}$

F esg ${ }^{\text {ts }} \quad 6 \mathrm{~d} 29^{\circ} \mathrm{C} \quad \mathbf{G}_{\text {MARCM82B } 7 \text { dahs }}$
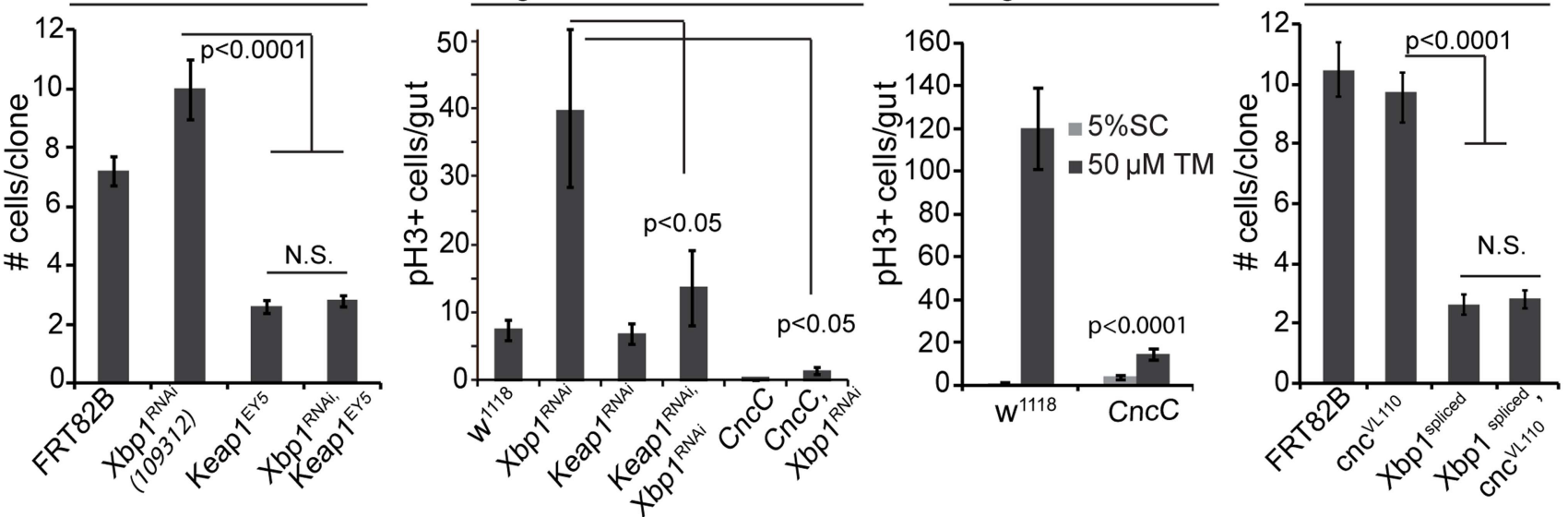
Figure 6. Xbp1 coordinates with Keap-CncC in ISC proliferation. (A) UPR ${ }^{\mathrm{ER}}$ influences $\mathrm{CncC}$ activity in ISCs/EBs (esgts). CncC activity assessed by bGal expression from the CncC reporter gstd1::IacZ. DAPI, blue; GFP, green; bGal and Hsc3 are shown as separate channels in white. Arrowheads point to individual ISCs/EBs. Quantification of relative bGal and Hsc3 staining shown in the right panel. Ratio of fluorescence intensities in ISCs/EBs and nearby ECs is shown. ISCs/EBs identified by GFP. P values from Student's T test. $N=3$. (B) Control of CncC activity by the UPR ${ }^{\mathrm{ER}}$ in response to tunicamycin treatment. CncC activity assessed by bGal expression from the CncC reporter gstd1::IacZ. DAPI, blue; GFP, green; bGal white, and shown as separate channel in white. Arrowheads point to individual ISCs/EBs. Quantification of bGal staining shown in the lower panel. Ratio of fluorescence intensities in ISCs/EBs and nearby ECs is shown. ISCs/EBs identified by GFP. P values from Student's T test. N=3. P values from Student's T test. N=5. (C) GFP-marked MARCM clones for Keap $1^{\mathrm{EY} 5}, \mathrm{Xbp} 1 \mathrm{RNAi}^{109312}$, Keap $1^{\mathrm{EY} 5} ; \mathrm{Xbp}^{\mathrm{RNNAi}}{ }^{109312}$ and wild-type control (Frt82B) at 7 days after heat shock. (D) Quantification of MARCM clone sizes at 7 days after heat shock. Number of clones examined: $n=89\left(\right.$ Frt82B); $n=56\left(X b p 1 R N A i^{109312}\right) ; n=32$ $\left(\right.$ Keap 1 $\left.{ }^{\mathrm{EY} 5}\right) ; n=88$ (Keap1 $^{\mathrm{EY} 5}, \mathrm{Xbp} 1^{\mathrm{RNAi1} 09312}$ ). Averages and SEM are shown. $\mathrm{P}$ values from Student's $\mathrm{T}$ test. (E) Increased CncC (by loss of Keap1 or over-expressing $\mathrm{CncC}$ ) inhibits ISC overproliferation induced by loss of Xbp1 in ISCs/EBs (esg ${ }^{\text {ts }}$ ). Averages and SEM are shown. P values from Student's $\mathrm{T}$ test. $\mathrm{N}=10$. (F) $\mathrm{CncC}$ in ISCs/EBs (esg::Gal4,tub::Gal80ts) inhibits tunicamycin-induced ISC proliferation. Averages and SEM are shown. $\mathrm{P}$ values from Student's T test. N>10. (G) Quantification of MARCM clone sizes for at 7 days after heat shock. Number of clones examined: $n=102$ (Frt82B); $n=99$ $\left(\mathrm{Xbp}^{\text {spliced }}\right) ; \mathrm{n}=23$ ( $\left.\mathrm{Cnc} \mathrm{CL}^{\mathrm{VL} 10}, \mathrm{Xbp}^{\text {spliced }}\right)$. Averages and SEM are shown. P values from Student's T test. See also Figure S4.

doi:10.1371/journal.pgen.1004568.g006

proliferative homeostasis of the fly intestine [32]. It will be intriguing to dissect the interaction between the UPR ${ }^{\mathrm{ER}}$ machinery, innate immune signaling in ECs, commensal homeostasis and stem cell function in detail, and we anticipate that these interactions have a significant effect on overall lifespan of the organism.

\section{Materials and Methods}

\section{Fly lines and husbandry}

Fly lines $w^{1118}$, frt82B, frt40A, UAS::nlsGFP, UAS::hsc3, UAS::Xbp1 ${ }^{\text {RNAi }}$ (TRip:HMS03015) were obtained from the Bloomington Drosophila stock center. The following RNAi lines were obtained from the Vienna Drosophila RNAi Center: UAS::Xbp1 ${ }^{\text {RNAi }}$ (v109312, v15347), UAS::Hrd1RNAi (v6870), UAS::bsk ${ }^{\mathrm{RNAi}}$.

The following fly lines were generously provided as indicated: y1w1; esg::Gal4/+ by Dr. S Hayashi; UAS::xbp1 ${ }^{\text {EP2112 }}$ by Dr. Kyoung Sang Cho; UAS::xbp1 ${ }^{\mathrm{d} 08698}$ by Dr. P. Fernandez-Funez; esg $^{\text {ts }}$ F/O by Dr. H. Jiang; $\mathrm{Su}(\mathrm{H})$ Gbe::Gal4 by Dr. S. Bray;

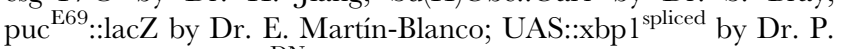
Domingos, UAS::bsk ${ }^{\text {DN }}$ by Dr. M. Mlodzik.

The Hrdl loss of function allele $\mathrm{Hrd1} 1^{\text {Delta }}$ was made by FRTmediated deletion of sequences between the Pbac insertion lines $\mathrm{Pbac}\{\mathrm{PB}\}$ sip3 c00467 and Pbac $\{\mathrm{PB}\}$ faf06363. hs-FLP was expressed in flies in which these Pbac insertions were in trans, deleting Hrdl and its nearby gene CG2126.

All flies were raised on yeast/molasses-based food at $25^{\circ} \mathrm{C}$ and $65 \%$ humidity on a $12 \mathrm{hr}$ light/dark cycle, unless otherwise noted.

For tunicamycin or paraquat exposure, flies were starved in empty vials for 6-8 hrs and fed with a $5 \%$ sucrose solution士 $50 \mu \mathrm{M}$ tunicamycin or $\pm 5 \mathrm{mM}$ paraquat for $24 \mathrm{hrs}$ followed by dissection in PBS.

For TARGET experiments, flies were raised at $18^{\circ} \mathrm{C}$ and shifted to $29^{\circ} \mathrm{C}$ at certain time points after eclosion. For MARCM clone induction, adult flies were aged for 1-2 days and then heat shocked at $37^{\circ} \mathrm{C}$ for $45 \mathrm{~min}$.

\section{Generation of Su (H)GBE-Gal80 transgenic flies}

The DNA fragment containing an enhancer of $\mathrm{Su}(\mathrm{H}) \mathrm{GBE}$ and a mini promoter of $\mathrm{HSP} 70$ was amplified from $\mathrm{Su}(\mathrm{H}) \mathrm{GBE}-\mathrm{Gal} 4$ [39] using PGR, with the following primers:

5'-AGTGAATTGAATTAGGGCTACTAGACTTG-3' (the 20th nucleotide " $T$ " is replaced by " $A$ " to eliminate the endogenous XbaI site).

5'-AGTTGTAGATCATGATGGGGGGGCTCAGGAGGCTTGGTTCAAGCTTG-3' (a NotI site was introduced in this primer).

The amplified DNA was cut and ligated into EcoRI and XbaI digested pCasper-Tub-Gal80 [1-7,59] to produce the construct
pCasper-Su(H)GBE. Then the DNA fragment containing Gal80 and Sv40 polyA was cut from pCasper-Tub-Gal80 at the NotI and XhoI sites, and ligated into NotI- and XhoI-digested pCasper$\mathrm{Su}(\mathrm{H}) \mathrm{GBE}$ to produce the $\mathrm{Su}(\mathrm{H}) \mathrm{GBE}-\mathrm{Gal} 80$ construct.

The construct was sequenced, purified, and microinjected into embryos using the standard method.

\section{Immunostaining and microscopy}

Guts were dissected in PBS, fixed for $45 \mathrm{~min}$ at room temperature in $100 \mathrm{mM}$ glutamic acid, $25 \mathrm{mM} \mathrm{KCl,} 20 \mathrm{mM}$ $\mathrm{MgSO}_{4}, 4 \mathrm{mM}$ sodium phosphate, $1 \mathrm{mM} \mathrm{MgCl}_{2}$, and $4 \%$ formaldehyde, washed for $1 \mathrm{hr}$, and incubated with primary antibodies and second antibodies in washing buffer (PBS, 0.5\% BSA, $0.1 \%$ Triton X-100).

The following primary antibodies were used:, guinea-pig antihsc3 antibody antibody [60] (1:150), mouse anti-Delta (Developmental Studies Hybridoma Bank, 1:100), rat anti-Delta (gift from Dr. MD Rand, University of Rochester, 1:1000); rabbit anti-PH3 (phosphorylated histone H3, Upstate, 1:1000), mouse anti- $\beta$-galactosidase (Developmental Studies Hybridoma Bank, 1:500), rabbit anti- $\beta$-galactosidase (Cappel, 1:5000), rabbit antipeIF2 $\alpha$ antibody (Cell Signaling: 3597, 1:150), mouse anti-pJNK antibody (Cell Signaling: 9255,1:150).

For Delta antibody staining, guts were fixed using a methanolheptane method as descried [61].

Fluorescent secondary antibodies were purchased from Jackson ImmunoResearch Laboratories. DNA was stained using DAPI. Confocal imaging was performed on a Zeiss LSM700 confocal microscope and processed using ImageJ and Adobe Illustrator.

\section{qRT-PCR analysis of gene expression}

Total RNA from young female samples were extracted using Trizol (Invitrogen) and cDNA was synthesized using Superscript III (Invitrogen). Real time RGR was performed on a Bio-Rad CFX96 detection system. Expression Values were normalized to RP49 expression levels. Primers included: total Xbpl transcripts (Forward:TGGGAGGAGAAAGTGCAAAG, Reverse:TCGGTTCTGTCTGTCAGGTC), Spliced Xbpl (Forward: ACGAACCTTGGATCTGCGG, Reverse:CGCGAAGCATGTCTTGTAGA), Hrdl (Forward:GCAGTTGGTCTTTGGCTTTG, Reverse: ATGGGCAGGGCGTATATTT), RP49(Forward:TCGTACGAGCTTCAAGATGAC, Reverse:CACGTTGTGCACGAGGAACT).

\section{ROS measurement via DHE}

ROS levels were measured as described before [19]. Briefly, guts were dissected in Schneider's medium, incubated in $30 \mu \mathrm{M}$ (Invitrogen) for $5 \mathrm{~min}$ at room temperature in the dark, washed twice and mounted to be imaged immediately. GFP expressed 


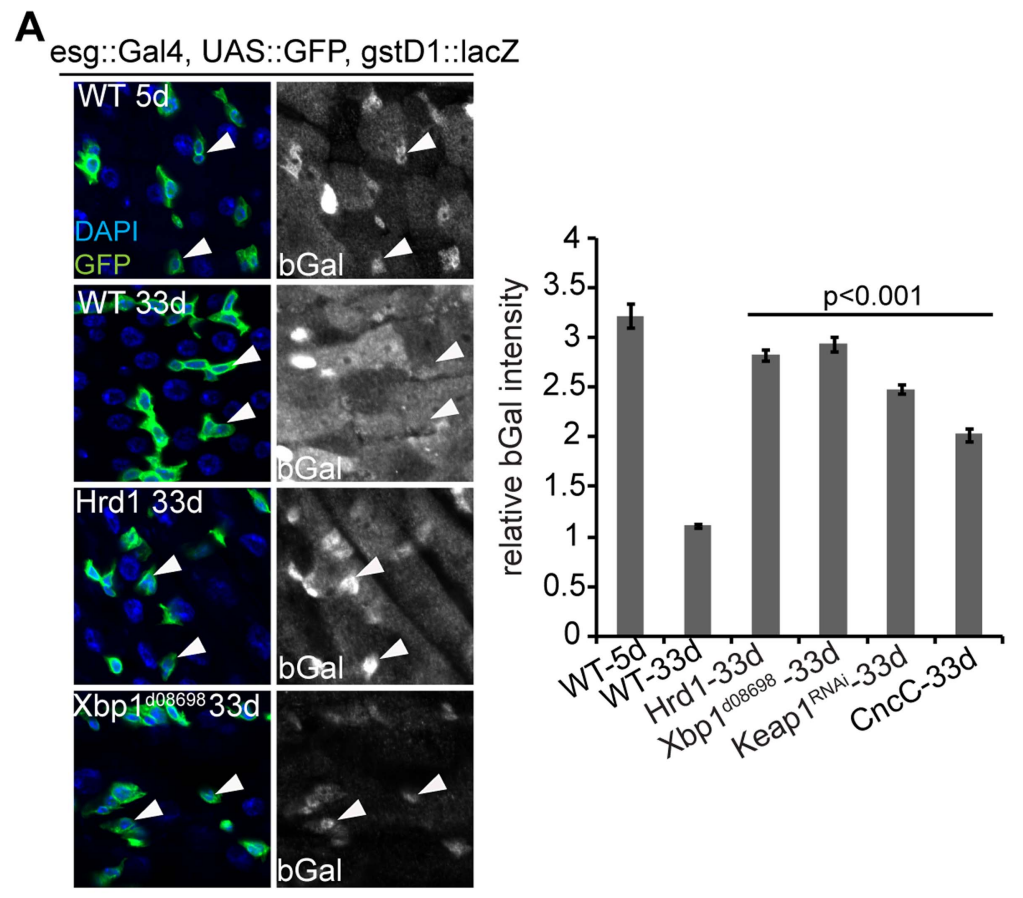

B esg::Gal4: UAS::GFP>

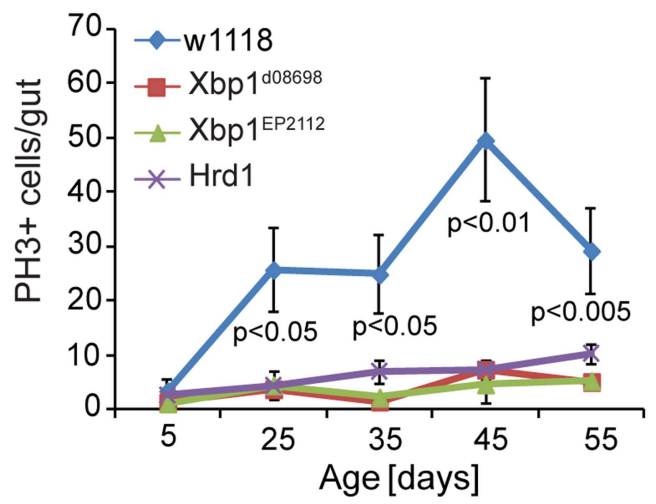

C

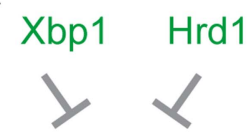

ER stress

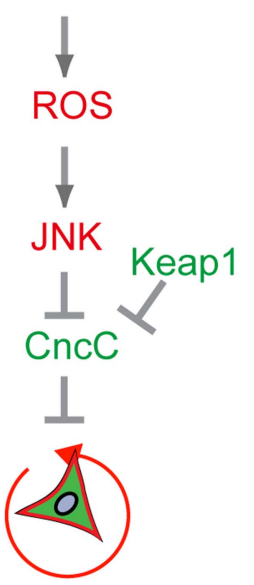

D

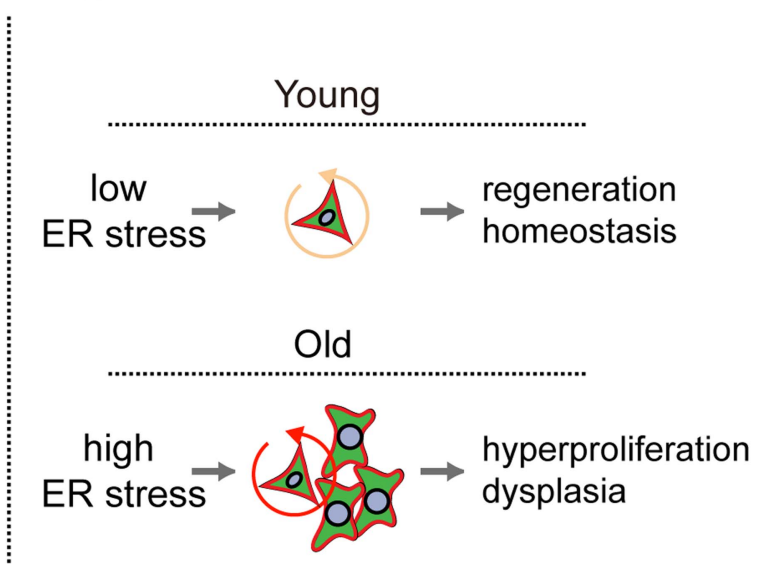


Figure 7. Improved ER quality control alleviates dysplasia of aging ISCs. (A) Intestines of wild-type flies stained with anti-bGal antibodies at 5 days or 33 days of age and of old flies over-expressing Hrd1 or Xbp1 (Xbp1 $1^{\text {d08698 }}$ ) in ISCs/EBs (esg::Gal4) and carrying the gstD1::lacZ reporter. Arrowheads point to ISCs/EBs (marked by GFP). Quantification of relative bGal staining shown in the right panel. Ratio of fluorescence intensity in ISCs/EBs and nearby ECs is shown. ISCs/EBs identified by GFP. P values from Student's T test. N $>=3$. (B) Quantification of mitotic figures in aging wild-type flies and in flies expressing Xbp1 (Xbp1 $1^{\text {do8698 }}$, Xbp ${ }^{\text {EP2112 }}$ ) and Hrd1 in ISCs/EBs (esg::Gal4, UAS::GFP). Averages and SEM are shown. P values from Student's T test, N>160 (from 4-5 guts each). N>20(5 d), N>20 (25 d), N>25 (35 d), N>7 (45 d), $n>14$ (55d)) (C) Model of UPR ${ }^{E R} / R O S$ signaling network regulating ISC proliferation, through ER stress is required for the induction of ISC proliferation. Inhibition of CncC activity by JNK in response to ER-induced ROS is further required to permit ISC regenerative responses. (D) Age-related loss of proliferative homeostasis as a consequence of increased ER stress. See also Figure S5.

doi:10.1371/journal.pgen.1004568.g007

under the control of esg::Gal4, $\mathrm{Su}(\mathrm{H}):: \mathrm{Gal} 80$ was used to identify ISGs and/or EBs.

\section{Supporting Information}

Figure S1 The UPR ${ }^{E R}$ is sufficient and required in ISGs to promote proliferation (related to Figure 1, 2, 3). (A) Knockdown $\mathrm{Xbpl}$ or Hrdl in $\operatorname{esg}^{\mathrm{ts}} \mathrm{F} / \mathrm{O}$ fly line (using esg::Gal4, tubGal80 ${ }^{\mathrm{ts}}$, UASFlp, act $>$ STOP $>$ Gal4) accelerates epithelial renewal. Representative images of wild-type fly and fly with loss of Xbpl are shown on the left. Quantification of clone sizes in the midgut area is shown on the right. (B) Representative images for MARCM clone sizes at 3 days after heat shock for Xbpl loss-of-function mutant $\left(\mathrm{Xbpl}^{\mathrm{k} 13803}\right)$ and wild-type control (Frt42D). (C) Representative images for MARCM clone sizes at 3 days after heat shock for knockdown of $\mathrm{Xbpl}$, of $\mathrm{Hrdl}$ and wild-type control (Frt40A). (D) Representative images for MARCM clone sizes at 3 days after heat shock for Hrdl loss-of-function mutant (Hrdl Delta) and wild-type control (Frt82B). (E) Representative images for MARCM clone sizes at 7 days after heat shock for Hrdl loss-offunction mutant $\left(\mathrm{Hrdl}^{\text {Delta }}\right)$ and wild-type control (Frt82B). (F) Representative images for MARCM clone sizes at 7 days after heat shock for spliced Xbpl, Xbpl ${ }^{\mathrm{d} 08698}$, Hrdl and wild-type control (Frt82B). (G) ISC-specific knockdown of Xbpl or Hrdl (using esg::Gal4, $\mathrm{Su}(\mathrm{H})$-Gbe::G80,tub::Gal80ts) induces ISG proliferation after 4 days of induction (shift to $29^{\circ} \mathrm{C}$ ). Averages and SEM are shown. $\mathrm{P}$ values from Student's T test, $\mathrm{N}>10 .(\mathrm{H})$ Quantification of $\mathrm{UPR}^{\mathrm{ER}}$ reporters shown in Fig. 1C, 1D, 1E. Relative fluorescence intensity is normalized to wild-type young guts. $\mathrm{P}$ values from Student's T test. $\mathrm{N}=6$ (hsc3 antibody staining); $\mathrm{N}=3$ (Xbplp reporter line).

(TIF)

Figure S2 Xbpls controls ISG proliferation in mitogenic conditions (related to Figure 3). (A) Spliced Xbpl inhibits Hep(JNKK)-induced ISC over-proliferation in ISCs/EBs (using esg::Gal4, tubG80 ${ }^{\text {ts }}$ ).(DNA: DAPI, blue; ISCs/EBs, GFP, ISCs: Delta staining). GFP and Delta channels are shown separately on the right. (B) Quantification of $\mathrm{pH} 3+$ cells in guts of 4 days and 14 days expressing Hep or InR or coexpressing Hep or InR with spliced Xbplin ISCis/EBs (using esg::Gal4, tubG80 ${ }^{\text {ts }}$ ). Averages and SEM are shown. $\mathrm{P}$ values from Student's Test, $\mathrm{N}=10$. (C) Deficiency of Notch-induced tumor formation is inhibited by expressed spliced Xbpl, but exacerbated by knockdown Xbpl in ISCs/EBs (using esg::Gal4, tubG80 ${ }^{\text {ts }}$ ). Representative images are shown. (DNA: DAPI blue; ISGs/EBs:GFP, green; ISCs: DI staining; EEs: Prospero staining). GFP, Delta and Prospero channels are shown separately on the right. (D) qRT-PCR validating effectiveness of RNAi and over-expression constructs for $\mathrm{UPR}^{\mathrm{ER}}$ components. Over-expression of Xbpl in Xbpl $\mathrm{X}^{\mathrm{d} 08698}$ or Xbp1 ${ }^{\mathrm{EP} 2112}$ animals crossed to actin::Gal4 was determined in whole flies. Expression of spliced Xbpl or $\mathrm{Hsc} 3$ using elav::Gal4, as well as knockdown of Xbpl (XbplRNAi ${ }^{15347}$ or XbplRNAi ${ }^{109312}$ ) or Hrdl using elav::Gal4 was determined in heads. $\mathrm{P}$ values from Student's Test, $\mathrm{N}>3$. Expression is relative to rp49. (E) Expression of UAS-linked transgenes by esg::Gal4 is limited to $\mathrm{Dl}+\mathrm{ISC}$ s by co-expression of $\mathrm{Gal} 80$ from the $\mathrm{Su}(\mathrm{H}) \mathrm{Gbe}$ promoter. Arrowheads point to Dl+ ISCs. DAPI blue, GFP green, Dl white.

(TIF)

Figure S3 $\mathrm{UPR}^{\mathrm{ER}}$-induced ISC proliferation is regulated by JNK activation (related to Figure 5). (A) JNK activation when Xbpl or Hrdl is knocked down in ISCs/EBs. Lines expressing dsRNA against Xbpl XbplRNAi $^{15347}, \mathrm{XbplRNAi}^{109312}$ and XbplRNAi ${ }^{\mathrm{HMS} 03015}$ ) or Hrdl were used. puc ${ }^{\mathrm{E} 69}:$ :lacZ reporter used to detect JNK activation. bGal is shown as separate channel in white. DAPI, blue; GFP, green; Armadillo, red. (B) Quantification of JNK activity (pJNK antibody and bGal staining for pucE69::lacZ) in Fig. 5A and Fig. S3. Relative fluorescence intensity is normalized to wild-type flies. P values from Student's T test. $\mathrm{N}=3$. (C) Repressing JNK activity (using Bsk ${ }^{\mathrm{RNAi}}$ ) inhibits ISC over-proliferation induced by loss of Xbpl in ISC/EBs (using $\mathrm{esg}^{\text {ts }}$ flip-out). Averages and SEM are shown. $\mathrm{P}$ values from Student's T test, $\mathrm{N}=10$.

(TIF)

Figure S4 Xbpl regulates ISC proliferation by regulating CncC activity (related to Figure 6). (A) Spliced Xbpl in ISC,s/EBs (using esg::Gal4, UAS-GFP, gstd1::lacZ, tubG80 ${ }^{\text {ts }}$ ) maintains high CncC activity in ISCs/EBs under ER stress. (DNA: DAPI blue; ISCs/ EBs: GFP). bGal channel is separately shown on the right. (B) Increased CncC activity inhibits ISC overproliferation induced by loss of Xbpl in esgtsF/O system (esg::Gal4, tubGal80ts, UASFlp, act $>$ STOP $>$ Gal4). Averages and SEM are shown. $\mathrm{P}$ values from Student's Test. $\mathrm{N}=10$.

(TIF)

Figure S5 Effects of $\mathrm{Hrdl}$ and $\mathrm{Keapl} / \mathrm{CncC}$ on ER stress in aging ISGs (related to Figure 7). (A) Old intestines (50 days) stained with anti-hsc3 antibody for wild-type and fly expressing Hrdlin ISCs/EBs (using esg::Gal4, UAS::GFP). Arrowheads point to the hsc3 staining in ISGs/EBs. (B) Old intestines (33 days) immunostained with anti-bGal and in wild-type fly (same as in Figure 7A) and fly expressing Keapl loss-of function, or overexpressing CncG. Arrowheads point to individual ISCs/EBs for bGal.

(TIF)

\section{Acknowledgments}

We thank Drs. Enrique Martín-Blanco, Huaqi Jiang, Kyoung Sang Cho, Marek Mlodzik, Pedro Fernandez-Funez, Pedro M Domingos, Sarah Bray, Shigeo Hayashi, and Steven X Hou for flies and reagents. We also thank Drs. M. Borch-Jensen and J. Karpac for critical comments on the manuscript.

\section{Author Contributions}

Conceived and designed the experiments: LW HJ. Performed the experiments: LW. Analyzed the data: LW HDR HJ. Contributed reagents/materials/analysis tools: LW XZ HDR HJ. Contributed to the writing of the manuscript: LW HDR HJ. 


\section{References}

1. Kaser A, Lee A-H, Franke A, Glickman JN, Zeissig S, et al. (2008) XBP1 Links ER Stress to Intestinal Inflammation and Confers Genetic Risk for Human Inflammatory Bowel Disease. Cell 134: 743-756. doi:10.1016/ j.cell.2008.07.021.

2. Niederreiter L, Fritz TMJ, Adolph TE, Krismer AM, Offner FA, et al. (2013) ER stress transcription factor Xbpl suppresses intestinal tumorigenesis and directs intestinal stem cells. Journal of Experimental Medicine 210: 2041-2056. doi: $10.1038 /$ nature 07589 .

3. Glimcher LH (2009) XBP1: the last two decades. Annals of the Rheumatic Diseases 69: i67-i71. doi:10.1136/ard.2009.119388.

4. Garrett WS, Gordon JI, Glimcher LH (2010) Homeostasis and inflammation in the intestine. Cell 140: 859-870. doi:10.1016/j.cell.2010.01.023.

5. Kaser A, Zeissig S, Blumberg RS (2010) Inflammatory bowel disease. Annu Rev Immunol 28: 573-621. doi:10.1146/annurev-immunol-030409-101225.

6. Kaser A, Flak MB, Tomczak MF, Blumberg RS (2011) The unfolded protein response and its role in intestinal homeostasis and inflammation. Experimental Cell Research 317: 2772-2779. doi:10.1016/j.yexcr.2011.07.008.

7. Adolph TE, Tomczak MF, Niederreiter L, Ko H-J, Böck J, et al. (2013) Paneth cells as a site of origin for intestinal inflammation. Nature 503: 272-276. doi:10.1038/nature12599.

8. Walter P, Ron D (2011) The unfolded protein response: from stress pathway to homeostatic regulation. Science 334: 1081-1086. doi:10.1126/science.1209038.

9. Schröder M, Kaufman RJ (2005) THE MAMMALIAN UNFOLDED PROTEIN RESPONSE. Annu Rev Biochem 74: 739-789. doi:10.1146/ annurev.biochem.73.011303.074134.

10. Travers KJ, Patil CK, Wodicka L, Lockhart DJ, Weissman JS, et al. (2000) Functional and genomic analyses reveal an essential coordination between the unfolded protein response and ER-associated degradation. Cell 101: 249-258.

11. Ryoo HD, Steller H (2007) Unfolded protein response in Drosophila: why another model can make it fly. Cell Cycle 6: 830-835.

12. Smith MH, Ploegh HL, Weissman JS (2011) Road to ruin: targeting proteins for degradation in the endoplasmic reticulum. Science 334: 1086-1090. doi:10.1126/science. 1209235

13. Frand AR, Kaiser CA (1999) Erolp oxidizes protein disulfide isomerase in a pathway for disulfide bond formation in the endoplasmic reticulum. Mol Cell 4: $469-477$.

14. Kim S, Sideris DP, Sevier CS, Kaiser CA (2012) Balanced Erol activation and inactivation establishes ER redox homeostasis. The Journal of Cell Biology 196: 713-725. doi:10.1083/jcb.201110090.

15. Gross E, Sevier CS, Heldman N, Vitu E, Bentzur M, et al. (2006) Generating disulfides enzymatically: reaction products and electron acceptors of the endoplasmic reticulum thiol oxidase Erolp. Proc Natl Acad Sci USA 103: 299-304. doi: 10.1073/pnas.0506448103.

16. Heijmans J, van Lidth de Jeude JF, Koo B-K, Rosekrans SL, Wielenga MCB, et al. (2013) ER Stress Causes Rapid Loss of Intestinal Epithelial Stemness through Activation of the Unfolded Protein Response. Cell reports 3: 1128-1139. doi:10.1016/j.celrep.2013.02.031.

17. Owusu-Ansah E, Banerjee U (2009) Reactive oxygen species prime Drosophila haematopoietic progenitors for differentiation. Nature 461: 537-541. doi:10.1038/nature08313.

18. Noble M, Smith J, Power J, Mayer-Proschel M (2003) Redox state as a central modulator of precursor cell function. Annals of the New York Academy of Sciences 991: 251-271.

19. Hochmuth CE, Biteau B, Bohmann D, Jasper H (2011) Redox regulation by Keap1 and Nrf2 controls intestinal stem cell proliferation in Drosophila. Cell Stem Cell 8: 188-199. doi:10.1016/j.stem.2010.12.006.

20. Tothova Z, Gilliland DG (2007) FoxO transcription factors and stem cell homeostasis: insights from the hematopoietic system. Cell Stem Cell 1: 140-152.

21. Luo B, Lee AS (2013) The critical roles of endoplasmic reticulum chaperones and unfolded protein response in tumorigenesis and anticancer therapies. Oncogene 32: 805-818. doi:10.1038/onc.2012.130.

22. Micchelli CA, Perrimon N (2006) Evidence that stem cells reside in the adult Drosophila midgut epithelium. Nature 439: 475-479. doi:10.1038/nature04371.

23. Ohlstein B, Spradling A (2006) The adult Drosophila posterior midgut is maintained by pluripotent stem cells. Nature 439: 470-474. doi:10.1038/ nature 04333 .

24. Biteau B, Hochmuth CE, Jasper H (2011) Maintaining tissue homeostasis: dynamic control of somatic stem cell activity. Cell Stem Cell 9: 402-411. doi:10.1016/j.stem.2011.10.004

25. Buchon N, Broderick NA, Lemaitre B (2013) Gut homeostasis in a microbial world: insights from Drosophila melanogaster. Nature reviews Microbiology 11: 615-626. doi: $10.1038 /$ nrmicro3074.

26. Cullinan SB, Diehl JA (2006) Coordination of ER and oxidative stress signaling: the PERK/Nrf2 signaling pathway. The international journal of biochemistry \& cell biology 38: 317-332.

27. Glover-Cutter KM, Lin S, Blackwell TK (2013) Integration of the Unfolded Protein and Oxidative Stress Responses through SKN-1/Nrf. PLoS Genet 9: e1003701. doi:10.1371/journal.pgen.1003701.

28. Biteau B, Hochmuth CE, Jasper H (2008) JNK activity in somatic stem cells causes loss of tissue homeostasis in the aging Drosophila gut. Cell Stem Cell 3: 442-455. doi:10.1016/j.stem.2008.07.024.
29. Choi NH, Kim JG, Yang DJ, Kim YS, Yoo MA (2008) Age-related changes in Drosophila midgut are associated with PVF2, a PDGF/VEGF-like growth factor. Aging Cell 7: 318-334. doi:10.1111/j.1474-9726.2008.00380.x.

30. Rera M, Clark RI, Walker DW (2012) Intestinal barrier dysfunction links metabolic and inflammatory markers of aging to death in Drosophila. Proceedings of the National Academy of Sciences 109: 21528-21533. doi:10.1073/pnas.1215849110.

31. Biteau B, Karpac J, Supoyo S, DeGennaro M, Lehmann R, et al. (2010) Lifespan extension by preserving proliferative homeostasis in Drosophila. PLoS Genet 6: e1001159.

32. Guo L, Karpac J, Tran SL, Jasper H (2014) PGRP-SC2 Promotes Gut Immune Homeostasis to Limit Commensal Dysbiosis and Extend Lifespan. Cell 156: 109-122. doi:10.1016/j.cell.2013.12.018.

33. Ryoo HD, Li J, Kang M-J (2013) Drosophila XBP1 expression reporter marks cells under endoplasmic reticulum stress and with high protein secretory load. PLoS ONE 8: e75774. doi:10.1371/journal.pone.0075774.

34. Sone M, Zeng X, Larese J, Ryoo HD (2013) A modified UPR stress sensing system reveals a novel tissue distribution of IRE1/XBP1 activity during normal Drosophila development. Cell Stress Chaperones 18: 307-319. doi:10.1007/ s12192-012-0383-x.

35. Jiang H, Patel PH, Kohlmaier A, Grenley MO, McEwen DG, et al. (2009) Cytokine/Jak/Stat signaling mediates regeneration and homeostasis in the Drosophila midgut. Cell 137: 1343-1355. doi:10.1016/j.cell.2009.05.014.

36. Ohlstein B, Spradling A (2007) Multipotent Drosophila intestinal stem cells specify daughter cell fates by differential notch signaling. Science 315: 988-992. doi: $10.1126 /$ science. 1136606 .

37. Casas-Tinto S, Zhang Y, Sanchez-Garcia J, Gomez-Velazquez M, RinconLimas DE, et al. (2011) The ER stress factor XBPls prevents amyloid-beta neurotoxicity. Human Molecular Genetics 20: 2144-2160. doi:10.1093/hmg/ ddr 100 .

38. Bordallo J, Plemper RK, Finger A, Wolf DH (1998) Der3p/Hrdlp is required for endoplasmic reticulum-associated degradation of misfolded lumenal and integral membrane proteins. Molecular biology of the cell 9: 209-222.

39. Zeng X, Chauhan C, Hou SX (2010) Characterization of midgut stem cell- and enteroblast-specific Gal4 lines in drosophila. Genesis 48: 607-611. doi:10.1002/ dvg.20661.

40. Luo L (2007) Fly MARCM and mouse MADM: genetic methods of labeling and manipulating single neurons. Brain Res Rev 55: 220-227. doi:10.1016/ j.brainresrev.2007.01.012.

41. Choi G, Park SH, Hwang S, Han SY, Hong YK, et al. (n.d.) Interference in $x b p 1$ gene expression induces defective cell differentiation and sensory organ development in Drosophila. Genes \& Genomics 32: 233-238. doi:10.1007/ s13258-010-0002-0.

42. Kang M-J, Ryoo HD (2009) Suppression of retinal degeneration in Drosophila by stimulation of ER-associated degradation. Proceedings of the National Academy of Sciences 106: 17043-17048. doi:10.1073/pnas.0905566106.

43. Dorner AJ, Bole DG, Kaufman RJ (1987) The relationship of N-linked glycosylation and heavy chain-binding protein association with the secretion of glycoproteins. The Journal of cell biology 105: 2665-2674.

44. Biteau B, Karpac J, Hwangbo D, Jasper H (2010) Regulation of Drosophila lifespan by JNK signaling. Exp Gerontol 46: 349-354. doi:10.1016/j.exger.2010.11.003.

45. Kapuria S, Karpac J, Biteau B, Hwangbo D, Jasper H (2012) Notch-mediated suppression of TSC2 expression regulates cell differentiation in the Drosophila intestinal stem cell lineage. PLoS Genet 8: e1003045.

46. Amcheslavsky A, Jiang J, Ip YT (2009) Tissue damage-induced intestinal stem cell division in Drosophila. Cell Stem Cell 4: 49-61. doi:10.1016/ j.stem.2008.10.016.

47. Harding HP, Zhang Y, Zeng H, Novoa I, Lu PD, et al. (2003) An integrated stress response regulates amino acid metabolism and resistance to oxidative stress. Mol Cell 11: 619-633.

48. Sevier CS, Kaiser CA (2008) Erol and redox homeostasis in the endoplasmic reticulum. Biochimica et biophysica acta 1783: 549-556. doi:10.1016/ j.bbamcr.2007.12.011.

49. Tu BP, Weissman JS (2004) Oxidative protein folding in eukaryotes: mechanisms and consequences. The Journal of cell biology 164: 341-346. doi:10.1083/jcb.200311055.

50. DeGennaro M, Hurd TR, Siekhaus DE, Biteau B, Jasper H, et al. (2011) Peroxiredoxin stabilization of DE-cadherin promotes primordial germ cell adhesion. Dev Cell 20: 233-243. doi:10.1016/j.devcel.2010.12.007.

51. Sykiotis GP, Bohmann D (2008) Keap 1/Nrf2 signaling regulates oxidative stress tolerance and lifespan in Drosophila. Dev Cell 14: 76-85. doi:10.1016/ j.devcel.2007.12.002

52. Biteau B, Jasper H (2011) EGF signaling regulates the proliferation of intestinal stem cells in Drosophila. Development 138: 1045-1055. doi:10.1242/ dev.056671.

53. Henis-Korenblit S, Zhang P, Hansen M, McCormick M, Lee SJ, et al. (2010) Insulin/IGF-1 signaling mutants reprogram ER stress response regulators to promote longevity. Proceedings of the National Academy of Sciences 107: 97309735. doi:10.1073/pnas.1002575107. 
54. Taylor RC, Dillin A (2013) XBP-1 is a cell-nonautonomous regulator of stress resistance and longevity. Cell 153: 1435-1447. doi:10.1016/j.cell.2013.05.042.

55. Kourtis N, Tavernarakis N (2011) Cellular stress response pathways and ageing: intricate molecular relationships. EMBO J 30: 2520-2531. doi:10.1038/ emboj.2011.162.

56. Taylor RC, Dillin A (2011) Aging as an event of proteostasis collapse. Cold Spring Harbor perspectives in biology 3. doi:10.1101/cshperspect.a004440.

57. Grootjans J, Hodin CM, de Haan J-J, Derikx JPM, Rouschop KMA, et al. (2011) Level of activation of the unfolded protein response correlates with Paneth cell apoptosis in human small intestine exposed to ischemia/reperfusion. Gastroenterology 140: 529-539.e3. doi:10.1053/j.gastro.2010.10.040.
58. Richardson CE, Kooistra T, Kim DH (2010) An essential role for XBP-1 in host protection against immune activation in C. elegans. Nature 463: 1092-1095. doi: $10.1038 /$ nature 08762 .

59. Lee T, Luo L (2001) Mosaic analysis with a repressible cell marker (MARCM) for Drosophila neural development. Trends Neurosci 24: 251-254.

60. Ryoo HD, Domingos PM, Kang M-J, Steller H (2006) Unfolded protein response in a Drosophila model for retinal degeneration. EMBO J 26: 242-252. doi:10.1038/sj.emboj.7601477.

61. Lin G, Xu N, Xi R (2008) Paracrine Wingless signalling controls self-renewal of Drosophila intestinal stem cells. Nature 455: 1119-1123. doi:10.1038/ nature07329. 